\title{
THEOREM PROVING FOR PRENEX GÖDEL LOGIC WITH $\triangle$ : CHECKING VALIDITY AND UNSATISFIABILITY
}

\author{
MATTHIAS BAAZ $^{a}$, AGATA CIABATTONI ${ }^{b}$, AND CHRISTIAN G. FERMÜLLER $^{c}$ \\ ${ }^{a}$ Department of Discrete Mathematics and Geometry, TU Vienna \\ e-mail address: baaz@logic.at \\ ${ }^{b, c}$ Department of Computer Languages, TU Vienna \\ e-mail address: \{agata,chrisf\}@logic.at
}

\begin{abstract}
First-order Gödel logic with the projection operator $\triangle\left(\mathbf{G}_{\infty}^{\triangle}\right)$ is an important many-valued as well as intermediate logic. In contrast to classical logic, the validity and the satisfiability problems of $\mathbf{G}_{\infty}^{\Delta}$ are not directly dual to each other. We nevertheless provide a uniform, computational treatment of both problems for prenex formulas by describing appropriate translations into sets of order clauses that can be subjected to chaining resolution. For validity a version of Herbrand's Theorem allows us to show the soundness of standard Skolemization. For satisfiability the translation involves a novel, extended Skolemization method.
\end{abstract}

\section{INTRODUCTION}

In classical logic efficient, resolution based theorem proving is a two step process. To prove the validity of an arbitrary first-order formula $F$ we first translate its negation into a Skolemized, purely universal conjunctive normal form of $\neg F$. This normal form directly corresponds to "logic free" syntax, namely to a set of clauses $c l(\neg F)$ that can be subjected to Robinson's celebrated resolution mechanism to test for unsatisfiability. The well attested efficiency of resolution, compared to other proof search methods, is due to the combination of the unification principle (the existence of most general unifiers) and simple atomic cuts as the only inference steps. The original formula $F$ is valid if and only if the empty clause, representing contradiction, is derivable from $\operatorname{cl}(\neg F)$ in this manner.

Note that in classical logic testing the validity of $F$ is equivalent to testing the unsatisfiability of $\neg F$. This duality is lost in the logic $\mathbf{G}_{\infty}^{\triangle}$ that we will consider here, namely Gödel logic enriched by the projection operator $\triangle[2]$. There are indeed $\mathbf{G}_{\infty}^{\triangle}$-formulas $F$ that are unsatisfiable, in the sense that there is no interpretation in $\mathbf{G}_{\infty}^{\triangle}$ that assigns the

1998 ACM Subject Classification: F.4.1.

Key words and phrases: Gödel logic, $\triangle$ modality, theorem proving, fuzzy logic, Skolemization, Herbrand's theorem, resolution, chaining calculus.

${ }^{a, b, c}$ Partly supported by FWF grant P22416, WWTF grant WWTF016, FWF START Y544-N23, and Eurocores-ESF/FWF grant 1143-G15 (LogICCC-LoMoReVI). 
designated truth value 1 to $F$, and nevertheless $\neg F$ is not valid in $\mathbf{G}_{\infty}^{\triangle}$. The importance of Gödel logic is emphasized by the fact that it naturally turns up in a number of different contexts; among them fuzzy logic [18, intermediate logics [28], the provability logic of Heyting arithmetic [29] and logic programming [24. In these contexts, both the validity problem and the satisfiability problem are of interest. In particular the latter is often crucial for applications, for instance to detect inconsistencies in the knowledge base of fuzzy rule-based systems [13]. The operator $\triangle$, which maps $\triangle G$ to the designated truth value 1 if the value of $G$ equals 1 , and to 0 otherwise, greatly increases the expressive power of Gödel logic and its applicability. However, it renders the resulting logic more complicated. For instance, checking whether a formula of $\mathbf{G}_{\infty}^{\triangle}$ is valid or satisfiable is undecidable already in the prenex and monadic case, i.e. when considering formulas with only unary predicates and no function symbols where a string of quantifiers precedes a quantifier free part; in contrast, without $\triangle$ satisfiability for prenex formulas is decidable, see [5, 3].

Our aim is to provide a uniform treatment of the validity and the satisfiability problem for first-order $\mathbf{G}_{\infty}^{\triangle}$ in as close analogy to classical logic as possible.

In contrast to propositional Gödel logic with or without $\triangle$ (e.g. [17, 22]), efficient automated theorem proving at the first-order level seems to be beyond the current state of the art, if possible at all. Thus it is reasonable to consider appropriate non-trivial fragments. Here we focus on the prenex fragment of $\mathbf{G}_{\infty}^{\triangle}$. We describe an efficient translation of such formulas into sets of order clauses that can then be subjected to chaining resolution [12, 11] to test for unsatisfiability. A central challenge here is to prove the soundness of (appropriate versions of) Skolemization.

The results below mainly bring together and round off what we have presented in preliminary form in two conference papers, 3] and [8]. The current paper is organized as follows. Section 2 provides formal definitions and basic facts about $\mathbf{G}_{\infty} \Delta$. Section 3 is devoted to Skolemization for prenex $\mathbf{G}_{\infty}^{\Delta}$. This requires a separate and different treatment of the validity and the satisfiability case. In Subsection 3.1 we prove that the standard Skolemization method that replaces strong quantifier occurrences by newly introduced Skolem terms preserves validity for prenex formulas in $\mathbf{G}_{\infty} \Delta$. The proof involves a version of Herbrand's theorem as well as a rather general statement about "reverse Skolemization", which is of independent interest. For testing (un-)satisfiability we cannot proceed as in classical logic, but rather introduce a novel extended form of Skolemization that, in addition to replacing weak quantifier occurrences by Skolem terms, introduces a fresh monadic predicate symbol. In Section 4 we demonstrate that the results of Section 3 enable a translation of prenex $\mathbf{G}_{\infty}^{\triangle}$-formulas into corresponding clause forms of a particular kind, namely order clauses that are to be interpreted in the theory of dense linear orders with endpoints. To achieve a reasonably efficient translation process we use definitional normal forms that introduce new predicate symbols for non-atomic subformulas. We show that both the validity and the satisfiability problem for prenex $\mathbf{G}_{\infty}^{\triangle}$ can be reduced to proving (un-)satisfiability of corresponding sets of order clauses. For the final step of theorem proving we rely on known results about so-called ordered chaining resolution [11, 12]. To render the paper self-contained we will explicitly state in Subsection 4.2 which inference rules and corresponding soundness and completeness result are needed in our specific case. 


\section{GÖDEL LOGIC WITH $\triangle$}

First-order Gödel logic $\mathbf{G}_{\infty}$, sometimes also called intuitionistic fuzzy logic [28] or Dummett's LC (eg. in [1, 16], referring to [15]), arises from intuitionistic logic by adding the axiom of linearity $(P \rightarrow Q) \vee(Q \rightarrow P)$ and the axiom $\forall x\left(P(x) \vee Q^{(x)}\right) \rightarrow(\forall x P(x)) \vee Q^{(x)}$ $\left(\vee\right.$-shift), where the notation $A^{(x)}$ indicates that there is no free occurrence of $x$ in $A$.

Semantically Gödel logic can be viewed as an infinite-valued logic where the real interval $[0,1]$ is taken as the set of truth values 1 An interpretation I consists of a non-empty domain $D$ and a valuation $v_{I}$ that maps constant symbols and object variables to elements of $D$ and $n$-ary function symbols to functions from $D^{n}$ into $D ; v_{I}$ extends in the usual way to a function mapping all terms of the language to an element of the domain. Moreover, every $n$-ary predicate symbol $p$ is mapped to a function $v_{I}(p)$ of type $D^{n} \mapsto[0,1]$. The truth-value of an atomic formula $($ atom $) p\left(t_{1}, \ldots, t_{n}\right)$ is defined as

$$
\left\|p\left(t_{1}, \ldots, t_{n}\right)\right\|_{I}=v_{I}(p)\left(v_{I}\left(t_{1}\right), \ldots, v_{I}\left(t_{n}\right)\right) .
$$

For the truth constants $\perp$ and $\top$ we have $\|\perp\|_{I}=0$ and $\|\top\|_{I}=1$.

The semantics of propositional connectives is given by

$$
\begin{array}{r}
\|A \wedge B\|_{I}=\min \left(\|A\|_{I},\|B\|_{I}\right), \\
\|A \rightarrow B\|_{I}= \begin{cases}1 & \|A \vee B\|_{I}=\max \left(\|A\|_{I},\|B\|_{I}\right), \\
\|B\|_{I} & \text { otherwise. }\end{cases}
\end{array}
$$

Henceforth we will consider the following abbreviations: $\neg A$ for $A \rightarrow \perp$ and $A \leftrightarrow B$ for $(A \rightarrow B) \wedge(B \rightarrow A)$. Therefore

$$
\|\neg A\|_{I}=\left\{\begin{array}{ll}
1 & \text { if }\|A\|_{I}=0 \\
0 & \text { otherwise }
\end{array} \quad\|A \leftrightarrow B\|_{I}= \begin{cases}1 & \text { if }\|A\|_{I}=\|B\|_{I} \\
\min \left(\|A\|_{I},\|B\|_{I}\right) & \text { otherwise. }\end{cases}\right.
$$

For quantification we define the distribution of a formula $A$ with respect to a free variable $x$ in an interpretation $I$ as $\operatorname{distr}_{I}(A(x))=\left\{\|A(x)\|_{I[d / x]} \mid d \in D\right\}$, where $I[d / x]$ denotes the interpretation that is exactly as $I$, except for insisting on $v_{I[d / x]}(x)=d$. Similarly we will use $I[\bar{d} / \bar{x}]$ for the interpretation arising from $I$ by assigning the domain element $d_{i}$ in $\bar{d}=d_{1}, \ldots, d_{n}$ to the variable $x_{i}$ in $\bar{x}=x_{1}, \ldots, x_{n}(1 \leq i \leq n)$. The universal and existential quantifiers correspond to the infimum and supremum, respectively, in the following sense:

$$
\|\forall x A(x)\|_{I}=\inf \operatorname{distr}_{I}(A(x)) \quad\|\exists x A(x)\|_{I}=\sup _{\operatorname{distr}}(A(x)) .
$$

2.1. The projection operator $\triangle$. Following [2], we enrich the language of $\mathbf{G}_{\infty}$ by adding the unary operator $\triangle$ with the following meaning:

$$
\|\triangle A\|_{I}= \begin{cases}1 & \text { if }\|A\|_{I}=1 \\ 0 & \text { otherwise. }\end{cases}
$$

The resulting logic - denoted as $\mathbf{G}_{\infty}^{\triangle}$-is strictly more expressive than $\mathbf{G}_{\infty}$. $\triangle$ allows to recover classical reasoning inside "fuzzy reasoning" in a very simple and natural manner: if all atoms are prefixed by $\triangle$ then $\mathbf{G}_{\infty}^{\triangle}$ coincides with classical logic. However, the

\footnotetext{
${ }^{1}$ For more information about Gödel logic - its winding history, importance, variants, alternative semantics and proof systems-see, e.g., [10, 4, 18, 27, 9].
} 
expressive power of $\triangle$ goes considerably beyond this. In particular, observe that in general $\triangle \exists x P(x) \rightarrow \exists x \triangle P(x)$ is not valid in $\mathbf{G}_{\infty}^{\triangle}$. (There are interpretations $I$ such that $\|\exists x P(x)\|_{I}=1$ although $\|P(x)\|_{I[d / x]}<1$ for all domain elements $d$.).

Definition 2.1. A formula $A$ is valid in $\mathbf{G}_{\infty}^{\triangle}$ (in symbols: $\models_{\mathbf{G}_{\infty}} A$ ) if $\|A\|_{I}=1$ for all interpretations $I . A$ is (1-)satisfiable (in symbols: $A \in 1 \mathrm{SAT}$ ) if $\|A\|_{I}=1$ for at least one interpretation $I$. Every such interpretation is called a model of $A$.

Remark 2.2. In $\mathbf{G}_{\infty}$ and $\mathbf{G}_{\infty}^{\triangle}$ validity as well as 1-satisfiability of a formula depend only on the relative order of the truth values of atomic formulas, but not on their specific values.

Remark 2.3. In contrast to classical logic, in $\mathbf{G}_{\infty}^{\triangle}$ validity and 1-(un)satisfiability are not dual concepts. 2 For instance, the formula $\neg(B \wedge \neg \triangle B)$ is not valid in $\mathbf{G}_{\infty}^{\triangle}$, although $B \wedge \neg \triangle B$ is unsatisfiable.

Since $\mathbf{G}_{\infty}^{\triangle}$ does not contain the identity predicate, the following version of the LöwenheimSkolem theorem is easily obtained, just like for classical or intuitionistic logic.

Proposition 2.4 ([10]). Every 1-satisfiable formula of $\mathbf{G}_{\infty}^{\triangle}$ has a model with countably infinite domain.

We list a few valid schemes of $\mathbf{G}_{\infty}^{\triangle}$ that will be used in later sections. Recall that the notation $A^{(x)}$ indicates that there is no free occurrence of $x$ in $A$.

Lemma 2.5. Let $A$ and $B$ be formulas of $\mathbf{G}_{\infty}^{\triangle}$.

(1) $\models_{\mathbf{G}_{\infty}} \triangle A \rightarrow A$

(2) $\models_{\mathbf{G}_{\infty} \Delta} \triangle(A \vee B) \leftrightarrow(\triangle A \vee \triangle B)$

$(3) \models_{\mathbf{G}_{\infty}} \Delta A(t)^{(x)} \rightarrow \exists x A(x)$

(4) $\models_{\mathbf{G}_{\infty}} \Delta \forall \forall A \leftrightarrow \forall x \triangle A$

$(5) \models_{\mathbf{G}_{\infty}} \forall x\left(A^{(x)} \rightarrow B(x)\right) \rightarrow\left(A^{(x)} \rightarrow \forall x B(x)\right)$

(6) $\left.\models_{\mathbf{G}_{\infty}} \forall x\left(B(x) \rightarrow A^{(x)}\right)\right) \rightarrow\left(\exists x B(x) \rightarrow A^{(x)}\right)$

2.2. Prenex Fragment. The prenex fragment of a logic is the set of all closed formulas of the form $\mathrm{Q}_{1} x_{1} \ldots \mathrm{Q}_{n} x_{n} P$, where $P$ is quantifier free and $\mathrm{Q}_{i} \in\{\forall, \exists\}$ for $1 \leq i \leq n$. Like in intuitionistic logic, also in Gödel logic (with or without $\triangle$ ) quantifiers cannot be shifted arbitrarily. Indeed the following classical quantifiers shifting laws do not hold:

- $\left(\forall x A \rightarrow B^{(x)}\right) \rightarrow \exists x\left(A \rightarrow B^{(x)}\right)$

- $\left(B^{(x)} \rightarrow \exists x A\right) \rightarrow \exists x\left(B^{(x)} \rightarrow A\right)$.

As a consequence arbitrary formulas are not equivalent to prenex formulas, in general. Nevertheless the prenex fragment of $\mathbf{G}_{\infty}^{\triangle}$ is quite expressive. By encoding the classical theory of two equivalence relations one can show that both the validity and the 1-satisfiability problem are undecidable, see [5, 3. In fact the prenex fragment of $\mathbf{G}_{\infty}^{\triangle}$ is undecidable already in the monadic case; i.e., when considering only unary predicates and no function symbols.

\footnotetext{
${ }^{2}$ The duality is preserved when considering the notion of positive satisfiability: a formula $A$ is positively satisfiable if there exists an interpretation $I$ such that $\|A\|_{I}>0$. However this notion is less natural than 1-satisfiability. In particular a formula can be positively satisfiable without admitting a model.
} 
This should be contrasted with the decidability of satisfiability of prenex monadic $\mathbf{G}_{\infty}[5]$ and of the validity problem for the prenex fragment of intuitionistic logic [14].

\section{Skolemization fOr PREnex $\mathbf{G}_{\infty}^{\triangle}$}

Loosely speaking, Skolemization with respect to validity is the replacement of strong quantifiers in a formula by fresh function symbols. Here positive occurrences of universal quantifiers and negative occurrences of existential quantifiers are called strong; the other quantifier occurrences are called weak. Skolemization with respect to satisfiability replaces the weak quantifiers in a formula instead. It is, of course, always possible to instantiate bound variables and delete corresponding quantifier occurrences. But the aim is to obtain a formula that is valid or 1-satisfiable if and only if the original formula is valid or 1-satisfiable, respectively. In classical logic this is achieved by replacing each variable occurrence $y$ that is bound by a strong (weak) quantifier by a Skolem term $f\left(x_{1}, \ldots, x_{n}\right)$ if $y$ is in the scope of the weak (strong) quantifier occurrences $\mathrm{Q}_{1} x_{1}, \ldots, \mathrm{Q}_{n} x_{n}$. We refer to this transformation as "standard Skolemization". We emphasize the fact that standard Skolemization is not sound for intuitionistic logic, not even for its prenex fragment (see, e.g., [25]).

Below we describe how and why Skolemization with respect to validity as well as to satisfiability can be achieved for the prenex fragment of $\mathbf{G}_{\infty}^{\triangle}$. More precisely, we show that the standard Skolemization method of classical logic also works for prenex formulas of $\mathbf{G}_{\infty}^{\triangle}$ in the context of validity, but fails with respect to 1-satisfiability. For the latter we present a novel Skolemization method where, in addition to Skolem terms that replace weak quantifier occurrences, a fresh monadic predicate symbol is introduced.

3.1. Validity. As in the case of intuitionistic logic, standard Skolemization does not preserve validity in $\mathbf{G}_{\infty}$ (and therefore in $\mathbf{G}_{\infty}^{\triangle}$ ). For instance the formula

$$
\forall x \neg \neg A(x) \rightarrow \neg \neg \forall x A(x)
$$

is not valid in $\mathbf{G}_{\propto} 3^{3}$ while its Skolemized version $\forall x \neg \neg A(x) \rightarrow \neg \neg A(c)$ is valid. In this section we show that the standard Skolemization method is nevertheless sound for the prenex fragment of $\mathbf{G}_{\infty}^{\triangle}$ with respect to validity.

Definition 3.1. Let $F=\mathrm{Q}_{1} y_{1} \ldots \mathrm{Q}_{n} y_{n} P$, with $\mathrm{Q}_{i} \in\{\forall, \exists\}$ be a (prenex) formula, where $P$ is quantifier free. The operator $\Phi(\cdot)$, to be applied from outside to inside, is defined as follows:

- $\Phi(\exists x A(x))=\exists x \Phi(A(x))$;

- $\Phi(\forall x A(x))=\Phi(A(f(\bar{y})))$, where $f$ is a fresh (Skolem) function symbol and $\bar{y}$ are the free variables in $\forall x A(x)$; if there are no such variables then $\Phi(\forall x A(x))=\Phi(A(c))$, for a fresh (Skolem) constant symbol $c$;

- $\Phi(A)=A$, if $A$ is quantifier free.

The Skolem form of $F$, denoted by $\exists \bar{x} P^{S}(\bar{x})$, is $\Phi(F)$.

\footnotetext{
${ }^{3}$ Any interpretation with domain $\left\{d_{1}, d_{2}, \ldots\right\}$ and $\inf _{i \geq 1}\|A(x)\|_{I\left[d_{i} / x\right]}=0$ but $\|A(x)\|_{I\left[d_{i} / x\right]}>0$, for all $d_{i}$, is not a model for the formula.

${ }^{4}$ The notation hides the fact that the Skolem form also depends on the quantifier prefix. However, below, the context will always provide the relevant information.
} 
Our aim is to provide a constructive proof that

$$
\models_{\mathbf{G}_{\infty}} \mathrm{Q}_{1} y_{1} \ldots \mathrm{Q}_{n} y_{n} P \quad \Leftrightarrow \quad \models_{\mathbf{G}_{\infty}} \exists \bar{x} P^{S}(\bar{x}) .
$$

The first step towards establishing the (more difficult) left-to-right direction of this equivalence is to prove Herbrand's theorem for prenex $\mathbf{G}_{\infty}^{\triangle}$ (see [3] or [10]).

Definition 3.2. Let $P$ be a formula. The Herbrand universe $U(P)$ of $P$ is the set of all variable free terms that can be constructed from the set of function symbols and constants occurring in $P$. To prevent $U(P)$ from being finite or empty we add a constant and a function symbol of positive arity if no such symbols appear in $P$.

The Herbrand base $\mathbf{B}(P)$ is the set of atoms constructed from the predicate symbols in $P$ and the terms of the Herbrand universe. A Herbrand expansion of $P$ is a disjunction of instances of $P$ where free variables are replaced with terms in $U(P)$.

The following lemma relies on the fact that the truth value of any formula $P$ of $\mathbf{G}_{\infty}^{\triangle}$ under a given interpretation only depends on the ordering of the respective values of atoms occurring in $P$.

Lemma 3.3. Let $P$ be a quantifier free formula of $\mathbf{G}_{\infty}^{\triangle}$. For every interpretation $I$ such that $\|P\|_{I}<1$ and every real number $c$, where $0<c<1$, there is an interpretation $I^{c}$ such that $\|P\|_{I^{c}} \leq c$.

Proof. Let $p_{1}, \ldots, p_{k}$ the atomic formulas in $P$ that do not evaluate to 0 or 1 under $I$. Without loss of generality, assume that $\left\|p_{1}\right\|_{I} \bowtie_{1} \ldots \rtimes_{k}\left\|p_{k}\right\|_{I}$ where each $\bowtie_{i}$ is either $=$ or $<. I^{c}$ is defined by assigning (possibly) new values $\left\|p_{i}\right\|_{I^{c}}$ to $p_{i}$ such that $\left\|p_{1}\right\|_{I^{c}} \bowtie_{1} \ldots \rtimes_{k}$ $\left\|p_{k}\right\|_{I^{c}}$ and $\left\|p_{k}\right\|_{I^{c}} \leq c$ and keeping the values 0 or 1 for the remaining atoms. The claim is easily proved by structural induction on $P$.

Theorem 3.4. Let $P$ be any quantifier-free formula of $\mathbf{G}_{\infty}$. If $\models_{\mathbf{G}_{\infty}} \exists \bar{x} P(\bar{x})$ then there exist tuples $\overline{t_{1}}, \ldots \overline{t_{n}}$ of terms in $U(P)$, such that $\models_{\mathbf{G}_{\infty}} \bigvee_{i=1}^{n} P\left(\overline{t_{i}}\right)$.

Proof. Let $A_{1}, A_{2}, \ldots$ be a non-repetitive enumeration of (the infinite set) $\mathbf{B}(P)$. We construct a "semantic tree" T; i.e., a systematic representation of all possible order types of interpretations of $A_{i}$. $\mathrm{T}$ is a rooted tree whose nodes appear at levels. Each node at level $\ell$ is labelled with an expression, called constraint, of the form

$$
c_{\ell}^{\pi} \stackrel{\text { def }}{=} 0 \bowtie_{0} A_{\pi(1)} \bowtie_{1} \ldots \bowtie_{\ell-1} A_{\pi(\ell)} \bowtie_{\ell} 1 \text {, }
$$

where $\bowtie$ is either $=$ or $<$ and $\pi$ is a permutation of $\{1, \ldots, \ell\}$. We say that an interpretation $I$ of $P(\bar{x})$ fulfills the constraint $c_{\ell}^{\pi}$ if

$$
0 \bowtie_{0}\left\|A_{\pi(1)}\right\|_{I} \bowtie_{1} \ldots \bowtie_{\ell-1}\left\|A_{\pi(\ell)}\right\|_{I} \bowtie_{\ell} 1
$$

holds. We say that the constraint $c_{\ell+1}^{\pi^{\prime}} \stackrel{\text { def }}{=} 0 \bowtie_{0} A_{\pi^{\prime}(1)} \bowtie_{1} \ldots \bowtie_{\ell} A_{\pi^{\prime}(\ell+1)} \bowtie_{\ell+1} 1$ extends $c_{\ell}^{\pi}$ if every interpretation fulfilling $c_{\ell+1}^{\pi^{\prime}}$ also fulfills $c_{\ell}^{\pi}$.

$\mathrm{T}$ is constructed inductively as follows:

- The root of $T$ is at level 0 and is labelled with the constraint $0<1$.

- Let $\nu$ be a node at level $\ell$ with label $c_{\ell}^{\pi}$.

$\left(*_{1}\right)$ Given $c_{\ell}^{\pi}$, if there is an instance $P(\bar{t})$ of $P(\bar{x})$, such that for every interpretation $I$ that fulfills $c_{\ell}^{\pi}$ we have $\|P(\bar{t})\|_{I}=1$, where the atoms of $P(\bar{t})$ are among $A_{1}, \ldots, A_{\ell}$, then $\nu$ is a leaf node of $\mathrm{T}$. 
Otherwise, for each constraint $c_{\ell+1}^{\pi^{\prime}}$ that extends $c_{\ell}^{\pi}$ a successor node $\nu^{\prime}$ labelled with this constraint is appended to $\nu$ (at level $\ell+1$ ).

By the definition of $\mathrm{T}$ the following holds:

$\left(*_{2}\right)$ For every interpretation $I$ of $\mathbf{B}(P)$ there is a branch of $\mathrm{T}$ such that $I$ fulfills all constraints at all nodes of this branch.

Two cases are to be considered:

$\mathrm{T}$ is finite: Let $\nu_{1}, \ldots, \nu_{m}$ be the leaf nodes of $\mathrm{T}$. Then by $\left(*_{1}\right)$ and $\left(*_{2}\right)$ we obtain $\models_{\mathbf{G}_{\infty}} \bigvee_{i=1}^{m} P\left(\overline{t_{i}}\right)$, where $P\left(\overline{t_{i}}\right)$ is an instance of $P(\bar{x})$ such that $\left\|P\left(\overline{t_{i}}\right)\right\|_{I}=1$ for all interpretations $I$ that fulfill the constraint at $\nu_{i}$.

$\mathrm{T}$ is infinite: By König's lemma, $\mathrm{T}$ has an infinite branch. This implies that there is an interpretation $I$ such that $\left\|P\left(\overline{t_{i}}\right)\right\|_{I}<1$ for every tuple $\overline{t_{i}}$ of terms of $U(P)$. By Lemma 3.3 there is an interpretation $J$ satisfying all constraints in the branch, such that $\left\|P\left(\overline{t_{i}}\right)\right\|_{J}<c<1$. Hence $\|\exists \bar{x} P(\bar{x})\|_{J}<1$, which contradicts the assumption that $\models_{\mathbf{G}_{\infty}} \exists \bar{x} P(\bar{x})$.

The following lemma states sufficient conditions for a logic to admit de-Skolemization (also known as reverse Skolemization). By this we mean the re-introduction of quantifiers in Herbrand expansions. These conditions are fulfilled by $\mathbf{G}_{\infty}^{\triangle}$ and indeed, by most of the fuzzy logics in the sense of [18]. Here, by a $\operatorname{logic} \mathbf{L}$ we mean a set of formulas that is closed under modus ponens. We call a formula $P$ valid in $\mathbf{L}$ (and write: $\models_{\mathbf{L}} P$ ) if $P \in \mathbf{L}$.

Lemma 3.5. Let $\mathbf{L}$ be a logic satisfying the following properties:

(1) $\models_{\mathbf{L}} P(t)^{(x)} \Rightarrow \models P(x)$

(2) $\models_{\mathbf{L}} Q \vee P \Rightarrow \models_{\mathbf{L}} P \vee Q$

$(3) \models_{\mathbf{L}}(Q \vee P) \vee R \Rightarrow \models_{\mathbf{L}} Q \vee(P \vee R)$

(4) $\models_{\mathbf{L}} Q \vee P \vee P \Rightarrow \models_{\mathbf{L}} Q \vee P$

$(5) \models_{\mathbf{L}} P(y) \Rightarrow \models_{\mathbf{L}} \forall x[P(x)]^{(y)}$

(6) $\models_{\mathbf{L}} P(t) \Rightarrow \models \mathbf{L} \exists x P(x)$

(7) $\models_{\mathbf{L}} \forall x\left(P(x) \vee Q^{(x)}\right) \Rightarrow \models_{\mathbf{L}}(\forall x P(x)) \vee Q^{(x)}$

(8) $\models_{\mathbf{L}} \exists x\left(P(x) \vee Q^{(x)}\right) \Rightarrow \models_{\mathbf{L}}(\exists x P(x)) \vee \exists x Q^{(x)}$.

Let $\exists \bar{x} P^{S}(\bar{x})$ be the Skolem form of $\mathrm{Q}_{1} z_{1} \ldots \mathrm{Q}_{n} z_{n} P\left(z_{1}, \ldots, z_{n}\right)$, where $\mathrm{Q}_{i} \in\{\forall, \exists\}$. For all tuples of terms $\overline{t_{1}}, \ldots, \overline{t_{m}}$ of the Herbrand universe of $P^{S}(\bar{x})$

$$
\models_{\mathbf{L}} \bigvee_{i=1}^{m} P^{S}\left(\overline{t_{i}}\right) \Rightarrow \models_{\mathbf{L}} \mathbf{Q}_{1} z_{1} \ldots \mathrm{Q}_{n} z_{n} P\left(z_{1}, \ldots, z_{n}\right) \text {. }
$$

Proof.5. Let $T_{S K}$ be the set of all instances in $\bigvee_{i=1}^{m} P^{S}\left(\overline{t_{i}}\right)$ of Skolem terms in $\exists \bar{x} P^{S}(\bar{x})$. We define the following order on $T_{S K}: s \prec t$ iff either $s$ is a proper subterm of $t$ or $s=f\left(t_{1}, \ldots, t_{a}\right)$ and $t=g\left(t_{1}^{\prime}, \ldots, t_{b}^{\prime}\right)$, where the Skolem term $f\left(x_{1}, \ldots, x_{a}\right)$ in $\exists \bar{x} P^{S}(\bar{x})$ replaces a variable $z_{i}$ in $\mathrm{Q}_{1} z_{1} \ldots \mathrm{Q}_{n} z_{n} P\left(z_{1}, \ldots, z_{n}\right)$ and $g\left(t_{1}^{\prime}, \ldots, t_{b}^{\prime}\right)$ replaces a variable $z_{j}$ in $\mathrm{Q}_{1} z_{1} \ldots \mathrm{Q}_{n} z_{n} P\left(z_{1}, \ldots, z_{n}\right)$ such that $i<j$. (Skolem constants are treated as 0-ary Skolem functions here.) As we will show below, this order guarantees that one can re-introduce universal quantifiers in $\bigvee_{i=1}^{m} P^{S}\left(\overline{t_{i}}\right)$ at the appropriate positions by replacing maximal terms at each corresponding step.

\footnotetext{
${ }^{5}$ Our de-Skolemization procedure follows the proof of the Second Epsilon Theorem by Hilbert and Bernays 21. A more detailed modern presentation of de-Skolemization for classical logic using Gentzen's sequent calculus can be found in [6]. Our task is to show that conditions 1-8 are sufficient to guarantee the soundness of the procedure and to correct an error in the proof of this lemma as it appeared in [3, 10].
} 
Starting with $\bigvee_{i=1}^{m} P^{S}\left(\overline{t_{i}}\right)$ and working from right to left with respect to the original quantifier prefix $\mathrm{Q}_{1} z_{1} \ldots \mathrm{Q}_{n} z_{n}$, we stepwise (re-)introduce quantifier occurrences at individual disjuncts using the algorithm below. We will use $\overline{\mathrm{Q}^{i}} P^{i}$ to denote a disjunct in the formula obtained at some stage of the procedure: the quantifier prefix $\overline{\mathrm{Q}^{i}}$ is either empty or else is $\mathrm{Q}_{k} z_{k} \ldots \mathrm{Q}_{n} z_{n}$ for some $1 \leq k \leq n$. Referring to $\overline{\mathrm{Q}^{i}} P^{i}$ we will use $\mathrm{Q}_{+}^{i} z$ to denote the quantifier occurrence immediately preceding $\overline{\mathrm{Q}^{i}}$ in $\mathrm{Q}_{1} z_{1} \ldots \mathrm{Q}_{n} z_{n}$. If $\overline{\mathrm{Q}^{i}}$ is empty then $\mathrm{Q}_{+}^{i} z=\mathrm{Q}_{n} z_{n}$. (If $\overline{\mathrm{Q}^{i}}$ is already the full prefix $\mathrm{Q}_{1} z_{1} \ldots \mathrm{Q}_{n} z_{n}$ then $\mathrm{Q}_{+}^{i}$ remains undefined.) Note that $\mathrm{Q}_{+}^{i} z$ denotes the quantifier occurrence that is to be introduced next at $\overline{\mathrm{Q}^{i}} P^{i}$.

Step 1: The following is repeated as long as possible:

Pick a disjunct $\overline{\mathrm{Q}^{i}} P^{i}$ in the current formula where $\mathrm{Q}_{+}^{i} z=\exists z_{k}$ for some $1 \leq k \leq n$. Replace $\overline{\mathrm{Q}^{i}} P^{i}(t)$ by $\exists z_{k} \overline{\mathrm{Q}^{i}} P^{i}\left(z_{k}\right)$, where $t$ is the term that occurs at those positions in $\overline{\mathrm{Q}^{i}} P^{i}$ where $z_{k}$ occurs in $P\left(z_{1}, \ldots, z_{n}\right)$.

Step 2: Remove all redundant copies of identical disjuncts, if any.

Step 3: Let $t$ be a maximal term (with respect to $\prec$ ) in $T_{S K}$ and let $\overline{\mathrm{Q}^{i}} P^{i}(t)$ be some disjunct of the current formula where $t$ occurs. $\mathrm{Q}_{+}^{i} z=\forall z_{k}$ for some $1 \leq k \leq n$. Replace $\overline{\mathrm{Q}^{i}} P^{i}(t)$ by $\forall z_{k} \overline{\mathrm{Q}^{i}} P^{i}\left(z_{k}\right)$. Goto step 1 .

We claim that the above algorithm converges at $\mathrm{Q}_{1} z_{1} \ldots \mathrm{Q}_{n} z_{n} P\left(z_{1}, \ldots, z_{n}\right)$ and that the validity of the current formula is preserved at each stage. To see why conditions 1-8 guarantee that this is the case we refer to the three steps separately.

Ad Step 1: Let $\bigvee_{i=1}^{\ell} \overline{\mathrm{Q}^{i}} P^{i}$ be the current formula. If there is no disjunct $\overline{\mathrm{Q}^{i}} P^{i}$ where $\mathrm{Q}_{+}^{i} z=\exists z_{k}$ for some $1 \leq k \leq n$ then step 1 is empty. Otherwise observe that

$$
\models_{\mathbf{L}} \bigvee_{j=1}^{j=i-1} \overline{\mathrm{Q}^{j}} P^{j} \vee \overline{\mathrm{Q}^{i}} P^{i}(t) \vee \bigvee_{j=i+1}^{j=\ell} \overline{\mathrm{Q}^{j}} P^{j}
$$

implies

$$
\models_{\mathbf{L}} \exists z_{k}\left(\bigvee_{j=1}^{j=i-1} \overline{\mathrm{Q}^{j}} P^{j} \vee \overline{\mathrm{Q}^{i}} P^{i}\left(z_{k}\right) \vee \bigvee_{j=i+1}^{j=\ell} \overline{\mathrm{Q}^{j}} P^{j}\right)
$$

by assumption 6 . Note that throughout the procedure the current formula remains closed, i.e. without free occurrences of variables. Therefore $z_{k}$ only occurs free in $\overline{\mathrm{Q}^{i}} P^{i}\left(z_{k}\right)$ and we can apply assumption 8 , combined with 2 and 3 , to obtain

$$
\models_{\mathbf{L}} \bigvee_{j=1}^{j=i-1} \overline{\mathrm{Q}^{j}} P^{j} \vee \exists z_{k} \overline{\mathrm{Q}^{i}} P^{i}\left(z_{k}\right) \vee \bigvee_{j=i+1}^{j=\ell} \overline{\mathrm{Q}^{j}} P^{j}
$$

as required.

Ad Step 2: Validity is preserved by assumptions 2, 3, and 4. Moreover, since the original quantifier occurrences $\mathrm{Q}_{k} z_{k}$ are re-introduced at their correct positions, the algorithm terminates with the original formula $\mathrm{Q}_{1} z_{1} \ldots \mathrm{Q}_{n} z_{n} P\left(z_{1}, \ldots, z_{n}\right)$, if steps 1 and 3 are sound.

Ad Step 3: We claim that (i) every maximal term $t$ occurs only in a single disjunct $\overline{\mathrm{Q}^{i}} P^{i}(t)$ of the current formula. Moreover, (ii) $\mathrm{Q}_{+}^{i} z=\forall z_{k}$ for some $1 \leq k \leq n$ and $t$ only occurs at positions where $z_{k}$ occurs in the original formula. Note that if these claims are true 
then

implies

$$
\models_{\mathbf{L}} \bigvee_{j=1}^{j=i-1} \overline{\mathrm{Q}^{j}} P^{j} \vee \overline{\mathrm{Q}^{i}} P^{i}(t) \vee \bigvee_{j=i+1}^{j=\ell} \overline{\mathrm{Q}^{j}} P^{j}
$$

$$
\models_{\mathbf{L}} \forall z_{k}\left(\bigvee_{j=1}^{j=i-1} \overline{\mathrm{Q}^{j}} P^{j} \vee \overline{\mathrm{Q}^{i}} P^{i}\left(z_{k}\right) \vee \bigvee_{j=i+1}^{j=\ell} \overline{\mathrm{Q}^{j}} P^{j}\right)
$$

by assumption 1 and 5 . This allows us to apply assumption 7, combined with 2 and 3 , to obtain

as required.

$$
\models_{\mathbf{L}} \bigvee_{j=1}^{j=i-1} \overline{\mathrm{Q}^{j}} P^{j} \vee \forall z_{k} \overline{\mathrm{Q}^{i}} P^{i}\left(z_{k}\right) \vee \bigvee_{j=i+1}^{j=\ell} \overline{\mathrm{Q}^{j}} P^{j}
$$

It remains to prove claims (i) and (ii). Since $t=f\left(t_{1}, \ldots, t_{n}\right)$ is an instance of a Skolem term $f\left(x_{1}, \ldots, x_{m}\right), t$ is connected to the universal variable $z_{j}$ in the original formula that has been replaced by $f\left(x_{1}, \ldots, x_{m}\right)$ in $\exists \bar{x} P^{S}(\bar{x})$. Let $\overline{\mathrm{Q}^{j}} P^{j}(t)$ be a disjunct of the current formula in which $t$ occurs. Because of step 1 we know that $\mathrm{Q}_{+}^{j} z=\forall z_{k}$ for some $1 \leq k \leq n$. Any position $p$ at which $t$ occurs in $\overline{\mathrm{Q}^{j}} P^{j}(t)$ is such that $z_{k}$ occurs in the original formula $\mathrm{Q}_{1} z_{1} \ldots \mathrm{Q}_{n} z_{n} P\left(z_{1}, \ldots, z_{n}\right)$ at $p$ for the following reasons. First, by maximality $t$ cannot be a subterm of any term that replaces one of $z_{1}, \ldots, z_{n}$ in the current formula. Moreover, by the definition of $\prec, t$ cannot replace any variable $z_{j}$ where $j<k$. This settles claim (ii).

Regarding claim (i), suppose that there were two disjuncts $\overline{\mathrm{Q}^{i}} P^{i}$ and $\overline{\mathrm{Q}^{j}} P^{j}$ in the current formula in which $t$ occurs. The maximality of $t=f\left(t_{1}, \ldots, t_{n}\right)$, claim (ii), and the fact that we have already re-introduced all quantifier occurrences to the right of $\forall z_{k}$ in $\mathrm{Q}_{1} z_{1} \ldots \mathrm{Q}_{n} z_{n}$ implies that the variables in the Skolem term $f\left(x_{1}, \ldots, x_{m}\right)$ correspond to precisely those variables in $\exists \bar{x} P^{S}(\bar{x})$ that are still instantiated by terms from the Herbrand universe in the current formula. But this means that at each position corresponding to an occurrence of $x_{i}(1 \leq i \leq m)$ in $\exists \bar{x} P^{S}(\bar{x})$ we find the same term $t_{i}$ in the current formula. Therefore $\overline{\mathrm{Q}^{i}} P^{i}$ and $\overline{\mathrm{Q}^{j}} P^{j}$ must be identical and thus have been contracted into a single disjunct at the preceding step 2 .

Corollary 3.6. Let $F=\bigwedge_{1 \leq i \leq m} A_{i}$ where $A_{i}$ are prenex formulas of $\mathbf{G}_{\infty}^{\triangle}$. Then

$$
\models_{\mathbf{G}_{\infty}} F \quad \Leftrightarrow \quad \models_{\mathbf{G}_{\infty}} \bigwedge_{1 \leq i \leq m} \exists \bar{x} A_{i}^{S}(\bar{x}) .
$$

Proof. $(\Rightarrow)$ Follows from the laws of quantification that hold in $\mathbf{G}_{\infty}^{\Delta}$.

$(\Leftarrow)$ Using Theorem 3.4 and Lemma 3.5 we argue as follows:

$$
\begin{array}{rlrl} 
& \models_{\mathbf{G}_{\infty}} \exists \bar{x} A_{i}^{S}(\bar{x}) \\
\Rightarrow & \models_{\mathbf{G}_{\infty}} \bigvee_{i=1}^{n} A_{i}^{S}\left(\overline{t_{i}}\right) \text { for appropriate } \overline{t_{1}}, \ldots, \overline{t_{m}} & \text { by Theorem } 3.4 \\
\Rightarrow \quad \models_{\mathbf{G}_{\infty}} A_{i} & \text { by Lemma } 3.5
\end{array}
$$

Since $\models_{\mathbf{G}_{\infty}} A$ iff for each $i=1, \ldots, m, \models_{\mathbf{G}_{\infty}} A_{i}$, this concludes the proof. 
3.2. Satisfiability. In contrast to the validity case, standard Skolemization for satisfiability (where weakly quantified variables are replaced by Skolem terms) does not preserve 1satisfiability, even for prenex formulas of $\mathbf{G}_{\infty}^{\triangle}$. This is due to the fact that to be 1-satisfiable, an existentially quantified formula does not need to evaluate to 1 for any of its instances. Rather, it is sufficient that the supremum of the distribution, i.e., of the truth values taken by the instances, is 1 . For instance, the following formula is 1-satisfiable:

$$
\exists x p(x) \wedge \forall y \neg \triangle p(y) .
$$

A model with domain $\left\{d_{i} \mid i \geq 1\right\}$ is obtained by setting $v_{I}(p)\left(d_{i}\right)=1-\frac{1}{i}$ for all $i \geq 1$. On the other hand the standard Skolemized form for this formula, $p(c) \wedge \forall y \neg \triangle p(y)$, is not 1-satisfiable. (The example can easily be made prenex by moving the universal quantifier to the front.)

Below we show that a Skolem form with respect to satisfiability for (conjunctions of) prenex formulas of $\mathbf{G}_{\infty}^{\triangle}$ can nevertheless be achieved by introducing an additional monadic predicate symbol. The resulting formulas will have no existential quantifier and will be 1-satisfiable if and only if the original formulas are. Given a prenex formula its Skolem form will be defined in two steps:

(1) We will first introduce a suitable formula, whose only existential quantifier binds the newly introduced monadic predicate, and which is 1-satisfiable if and only if the original formula is (Lemma 3.8).

(2) This existential quantifier is afterwards replaced by a universally quantified formula (Lemma 3.11).

Definition 3.7. Let $q$ be a new monadic predicate symbol. The operator $\Psi_{q}(\cdot)$, to be applied to prenex formulas from outside to inside, is defined by

- $\Psi_{q}(\forall x A(x))=\forall x \Psi_{q}(A(x))$;

- $\Psi_{q}(\exists x A(x))=\forall x\left(q(x) \rightarrow \Psi_{q}(A(f(x, \bar{y})))\right.$, where $f$ is a new (Skolem) function symbol and $\bar{y}$ are the free variables in $\exists x A(x)$;

- $\Psi_{q}(A)=A$, if $A$ is quantifier free.

The SAT-Skolem form $\operatorname{SKO}_{q}(A)$ of $A$ is obtained by moving all (universal) quantifiers in $\Psi_{q}(A)$ to the front and inserting one occurrence of $\triangle$ immediately after the quantifiers. More precisely,

$$
\mathrm{SKO}_{q}(A)=\forall \bar{x} \triangle\left(\Psi_{q}(A)^{-}\right)
$$

where $\bar{x}$ are the bounded variables in $\Psi_{q}(A)$, and $\Psi_{q}(A)^{-}$denotes $\Psi_{q}(A)$ after the removal of all quantifier occurrences.

Note that applying the operator $\mathrm{SKO}_{q}(\cdot)$ is not sufficient for our purpose, since $\mathrm{SKO}_{q}(P)$ is 1-satisfiable for all formulas $P$. For instance, $P=\exists x(\triangle A(x) \wedge \neg \triangle A(x))$ is not 1satisfiable, while $\mathrm{SKO}_{q}(P)=\forall x(q(x) \rightarrow(\triangle A(f(x)) \wedge \neg \triangle A(f(x)))$ is 1-satisfiable. (A model of the latter formula is obtained by setting $v_{I}(q)(d)=0$ for all $d$ in the domain $D$.) However $\mathrm{SKO}_{q}(\cdot)$ does preserve 1-(un)satisfiability when the condition $\sup \left\{v_{I}(q)(d) \mid d \in D\right\}=1$ is imposed in addition. As shown in the following theorem, this amounts to adding the formula $\exists x q(x)$ conjunctively to $\mathrm{SKO}_{q}(P)$. Henceforth we will slightly widen our focus by considering conjunctions of prenex formulas.

Lemma 3.8 (Step 1). Let $A_{1}, \ldots, A_{m}$ be prenex formulas. Then

$$
\left(\bigwedge_{1 \leq i \leq m} A_{i}\right) \in 1 \mathrm{SAT} \Longleftrightarrow\left(\exists x q(x) \wedge \bigwedge_{1 \leq i \leq m} \operatorname{SKO}_{q}\left(A_{i}\right)\right) \in 1 \mathrm{SAT} .
$$


Proof. Observe that $\mathrm{SKO}_{q}\left(A_{i}\right)$ is of the form

$$
\forall y_{1} \ldots \forall y_{k} \forall x_{1} \ldots \forall x_{n} \triangle\left(q\left(x_{1}\right) \rightarrow \ldots\left(q\left(x_{n}\right) \rightarrow A_{i}^{s k}\right) \ldots\right)
$$

where $A_{i}^{s k}$ denotes the quantifier free part of $A_{i}$ with existentially bound variables replaced by Skolem terms $f_{j}\left(x_{j}, \overline{y_{j}}\right)(1 \leq j \ldots n)$, as specified in Definition 3.7 .

$(\Leftarrow)$ We first show that

$$
(*) \models_{\mathbf{G}_{\infty}}\left(\exists x q(x) \wedge \mathrm{SKO}_{q}\left(A_{i}\right)\right) \rightarrow \forall y_{1} \ldots \forall y_{k} \exists v_{1} \ldots \exists v_{n} A_{i}^{s k}\left[f_{j}\left(x_{j}, \overline{y_{j}}\right) / f_{j}\left(v_{j}, \overline{y_{j}}\right)\right]
$$

where $v_{j}$ are fresh variables and $A_{i}^{s k}\left[f_{j}\left(x_{j}, \overline{y_{j}}\right) / f_{j}\left(v_{j}, \overline{y_{j}}\right)\right]$ stands for $A_{i}^{s k}$ in which the $x_{j}$ in Skolem terms are replaced by these new variables.

Since $\models_{\mathbf{G}_{\infty}} \triangle \forall x B \leftrightarrow \forall x \triangle B$ and $\models_{\mathbf{G}_{\infty}} \triangle B \rightarrow B$ for all formulas $B$ (Lemma2.5(1),(4)), we can remove the indicated occurrence of $\triangle$ in $\operatorname{SKO}_{q}\left(A_{i}\right)$. Then we use $\models_{\mathbf{G}_{\infty}} \forall x\left(B^{(x)} \rightarrow\right.$ $C(x)) \rightarrow\left(B^{(x)} \rightarrow \forall x C(x)\right)$ (Lemma 2.5(5)) to put all universally quantified variables $y_{j}$ immediately in front of $A_{i}^{s k}$ and $\models_{\mathbf{G}_{\infty}} A\left(f_{j}\left(x_{j}, \overline{y_{j}}\right)\right) \rightarrow \exists v_{j} A\left(f_{j}\left(v_{j}, \overline{y_{j}}\right)\right)$ (Lemma 2.5 (3)), where $v_{j}$ is a new variable. Finally by using Lemma 2.5(6) we move existential quantifiers immediately in front of all occurrences of $q$ to get

$\models_{\mathbf{G}_{\infty}} \operatorname{SKO}_{q}\left(A_{i}\right) \rightarrow\left(\exists x q(x) \rightarrow \ldots\left(\exists x q(x) \rightarrow \forall y_{1} \ldots \forall y_{k} \exists v_{1}, \ldots \exists v_{n} A_{i}^{s k}\left[f_{j}\left(x_{j}, \overline{y_{j}}\right) / f_{j}\left(v_{j}, \overline{y_{j}}\right)\right]\right) \ldots\right)$ from which $(*)$ follows straightforwardly. Therefore, if $\left(\exists x q(x) \wedge \mathrm{SKO}_{q}\left(A_{i}\right)\right) \in$ 1SAT then also $\forall y_{1} \ldots \forall y_{k}, \exists v_{1}, \ldots \exists v_{n} A_{i}^{s k}\left[f_{j}\left(x_{j}, \overline{y_{j}}\right) / f_{j}\left(v_{j}, \overline{y_{j}}\right)\right] \in 1 \mathrm{SAT}$. In fact, it is easy to transform a model of the latter formula into a model of $A_{i}$.

$(\Rightarrow)$ Suppose that the interpretation $I$ is a model for $A_{i}$ for $1 \leq i \leq m$. By Proposition 2.4 we can assume that $I$ has a countably infinite domain $D=\left\{d_{1}, d_{2}, \ldots\right\}$. To obtain a model $J$ with the same domain $D$ for the formula $\exists x q(x) \wedge \bigwedge_{1<i<m} \operatorname{SKO}_{q}\left(A_{i}\right)$ we have to augment $I$ by a suitable interpretation of $q$ and of the Skolem function symbols. In particular, to achieve $\|\exists x q(x)\|_{J}=1$ we assign $v_{J}(q)\left(d_{i}\right)=w_{i}$ in such a manner that $\sup _{i} w_{i}=1$, but $w_{i} \neq 1$ for all $i \geq 1$.

In interpreting the Skolem functions we have to make sure that for each step of the transformation $\Psi_{q}\left(B_{i}\right)$, replacing an existential quantifier in $\exists x B_{i}(x)$,

$$
\left\|q(x) \rightarrow \Psi_{q}\left(B_{i}(f(x, \bar{y}))\right)\right\|_{J[d / x, \bar{e} / \bar{y}]}=1,
$$

for all $d \in D$ and all $\bar{e} \in D^{n}$, where $n$ is the number of free variables in $\exists x B_{i}(x)$. To this aim we use the assumption that $\left\|\exists x B_{i}(x)\right\|_{I[\bar{e} / \bar{y}]}=1$. This means that for any $d \in D$ there is a further domain element $d^{\prime}$ such that $\|q(x)\|_{J[d / x]} \leq\left\|B_{i}(x)\right\|_{I\left[d^{\prime} / x, \bar{e} / \bar{y}\right]}$. We assign $v_{J}(f)(d, \bar{e})=d^{\prime}$. If there are no more existential quantifiers in $B_{i}$ then we are done, as $\Psi_{q}\left(B_{i}\right)=B_{i}$ and therefore $\left\|B_{i}(x)\right\|_{I\left[d^{\prime} / x, \bar{e} / \bar{y}\right]}=\left\|\Psi_{q}\left(B_{i}(f(x, \bar{y}))\right)\right\|_{J[d / x, \bar{e} / \bar{y}]}$. Otherwise we proceed by induction on the number of existential quantifiers replaced by applying $\Psi_{q}$, with (essentially) the presented argument as inductive step.

We will replace the newly introduced existential quantified formula $\exists x q(x)$ by a conjunction of suitable universal formulas. This way we will finally obtain a purely universal formula that is 1-satisfiable if and only if the original formula is 1-satisfiable. To this aim we first introduce a notation that will be useful also in the next section.

Definition 3.9. $A \unlhd B \stackrel{\text { def }}{=} \triangle(A \rightarrow B)$ and $A \triangleleft B \stackrel{\text { def }}{=} \neg \triangle(B \rightarrow A)$. 
It is straightforward to check that the suggestive symbols are justified by

$$
\|A \unlhd B\|_{I}=\left\{\begin{array}{ll}
1 & \text { if }\|A\|_{I} \leq\|B\|_{I} \\
0 & \text { otherwise }
\end{array} \quad \text { and } \quad\|A \triangleleft B\|_{I}= \begin{cases}1 & \text { if }\|A\|_{I}<\|B\|_{I} \\
0 & \text { otherwise. }\end{cases}\right.
$$

Definition 3.10. Let $A$ be a conjunction of prenex formulas of $\mathbf{G}_{\infty}^{\triangle}$ and $p_{1}, \ldots, p_{k}$ the predicate symbols occurring in $\mathrm{SKO}_{q}(A)$. (Note that $q \in\left\{p_{1}, \ldots, p_{k}\right\}$.)

$$
H_{\exists}(q, A) \stackrel{\text { def }}{=} \bigwedge_{1 \leq i \leq k} \forall \overline{y_{i}}\left(\top \unlhd p_{i}\left(\overline{y_{i}}\right) \vee p_{i}\left(\overline{y_{i}}\right) \triangleleft q\left(f_{p_{i}}\left(\overline{y_{i}}\right)\right)\right),
$$

where $\overline{y_{i}}$ is a sequence of fresh variables, according to the arity of $p_{i}$, and $f_{p_{i}}$ is a fresh function symbol of corresponding arity.

Lemma 3.11 (Step 2). Let $A=\bigwedge_{1 \leq i \leq m} A_{i}$ where $A_{1}, \ldots, A_{m}$ are prenex formulas. Then

$$
\left(\exists x q(x) \wedge \bigwedge_{1 \leq i \leq m} \operatorname{SKO}_{q}\left(A_{i}\right)\right) \in 1 \mathrm{SAT} \Longleftrightarrow\left(H_{\exists}(q, A) \wedge \bigwedge_{1 \leq i \leq m} \operatorname{SKO}_{q}\left(A_{i}\right)\right) \in 1 \mathrm{SAT} .
$$

Proof. For the whole proof let $p_{1}, \ldots, p_{k}$ be the predicate symbols occurring in $\operatorname{SKO}_{q}(A)$.

$(\Rightarrow)$ Let $I$ be a model of $\exists x q(x) \wedge \bigwedge_{1 \leq i \leq m} \operatorname{SKO}_{q}\left(A_{i}\right)$ with domain $D$. For every $\bar{d} \in D^{n}$, where $n$ is the arity of $p_{i}$ the following holds: either $\left\|p_{i}\left(\overline{y_{i}}\right)\right\|_{I\left[\bar{d} / \overline{y_{i}}\right]}=1$ or $\left\|p_{i}\left(\overline{y_{i}}\right)\right\|_{I\left[\bar{d} / \overline{y_{i}}\right]}<1$. In the former case the first disjunct $\top \unlhd p_{i}\left(\overline{y_{i}}\right)$ of the relevant conjunct in $H_{\exists}(q, A)$ evaluates to 1 . In the latter case, since we have $\|\exists x q(x)\|_{I}=1$, we can extend $I$ by a valuation function for the new function symbols $f_{p_{i}}$ in such a manner that $\left\|q\left(f_{p_{i}}\left(\overline{y_{i}}\right)\right)\right\|_{I\left[\bar{d} / \overline{y_{i}}\right]}>\left\|p_{i}\left(\overline{y_{i}}\right)\right\|_{I\left[\bar{d} / \overline{y_{i}}\right]}$ holds. But this implies that the second disjunct in $H_{\exists}(q, A)$ is evaluated to 1 .

$(\Leftarrow)$ Let $I$ be a model of $H_{\exists}(q, A) \wedge \bigwedge_{1 \leq i \leq m} \operatorname{SKO}_{q}\left(A_{i}\right)$ with domain $D$. If $\|\exists x q(x)\|_{I}=1$ then we are done. Otherwise, $\|q(x)\|_{I[d / x]}<1$ for all $d \in D$. Note that $q \in\left\{p_{1}, \ldots, p_{k}\right\}$ and therefore $\left\|H_{\exists}(q, A)\right\|_{I}=1$ implies that $\|q(x)\|_{I[d / x]}<\left\|q\left(f_{q}(x)\right)\right\|_{I[d / x]}$, for every $d \in D$, since $\|\top \unlhd q(x)\|_{I[d / x]}<1$. Consequently $\sup _{d \in D}\|q(x)\|_{I[d / x]}=v$ for some $v<1$, but nevertheless $\|q(x)\|_{I[d / x]} \neq v$ for all $d \in D .\left\|H_{\exists}(q, A)\right\|_{I}=1$ also implies that for every $\bar{d} \in D^{n}$, where $n$ is the arity of $p_{i}$, we have either $\left\|p_{i}\left(\overline{y_{i}}\right)\right\|_{I\left[\bar{d} / \overline{y_{i}}\right]}=1$ or $\left\|p_{i}\left(\overline{y_{i}}\right)\right\|_{I\left[\bar{d} / \overline{y_{i}}\right]}<v$. In other words: no atomic formula is assigned a value in the interval $[v, 1)$ by $I$. We may therefore define a new interpretation $J$ over the same domain $D$ by setting $\left\|p_{i}\left(\overline{y_{i}}\right)\right\|_{J\left[\bar{d} / \overline{y_{i}}\right]}=\left\|p_{i}\left(\overline{y_{i}}\right)\right\|_{I\left[\bar{d} / \overline{y_{i}}\right]}+(1-v)$, whenever $\left\|p_{i}\left(\overline{y_{i}}\right)\right\|_{I\left[\bar{d} / \overline{y_{i}}\right]} \neq 1$ and $\left\|p_{i}\left(\overline{y_{i}}\right)\right\|_{I\left[\bar{d} / \overline{y_{i}}\right]} \neq 0$. Otherwise the corresponding truth value remains the same, i.e., 1 or 0 , respectively.

It remains to show that $J$ is a model of $\exists x q(x) \wedge \bigwedge_{1<i<m} \operatorname{SKO}_{q}\left(A_{i}\right)$. By definition of $J$, $\sup _{d \in D}\|q(x)\|_{J[d / x]}=1$. To complete the argument remember that each $\operatorname{SKO}_{q}\left(A_{i}\right)$ is of the form $\forall \bar{x} \triangle B_{i}^{-}$where $B_{i}^{-}$is $\left(q\left(x_{1}\right) \rightarrow \ldots\left(q\left(x_{n}\right) \rightarrow A_{i}^{s k}\right) \ldots\right)$. Therefore $\left\|\operatorname{SKO}_{q}\left(A_{i}\right)\right\|_{I}=1$ implies that $\left\|B_{i}^{-}\right\|_{I[\bar{d} / \bar{x}]}=1$ for every appropriate tuple $\bar{d}$ of domain elements. This means that the evaluation reduces to that of a quantifier free formula of $\mathbf{G}_{\infty}^{\triangle}$. Now recall from Lemma 3.3 that whether a given interpretation $I$ satisfies a quantifier free formula only depends on the relative order of assigned truth values below 1 and above 0 , but not on their absolute values. Therefore, just like $I$, also $J$ is a model of $\operatorname{SKO}_{q}\left(A_{i}\right)$ for $1 \leq i \leq m$.

Example 3.12. Let $F=\exists x p(x) \wedge \forall y \neg \triangle p(y)$.

Step 1: by Lemma 3.8,

$$
F \in 1 \mathrm{SAT} \quad \text { if and only if } \exists x q(x) \wedge \mathrm{SKO}_{q}(\exists x p(x)) \wedge \forall y \neg \triangle p(y) \in 1 \mathrm{SAT},
$$


where $\mathrm{SKO}_{q}(\exists x p(x))=\forall x(q(x) \rightarrow p(f(x)))$.

Step 2: the existential quantifier is removed by translating $\exists x q(x)$ into $H_{\exists}(q, F)$ :

$$
\begin{aligned}
& \forall y_{1}\left(\top \unlhd q\left(y_{1}\right) \vee q\left(y_{1}\right) \triangleleft q\left(f_{q}\left(y_{1}\right)\right)\right) \\
\wedge & \forall y_{2}\left(\top \unlhd p\left(y_{2}\right) \vee p\left(y_{2}\right) \triangleleft q\left(f_{p}\left(y_{2}\right)\right)\right) \\
\wedge & \forall x(q(x) \rightarrow p(f(x))) .
\end{aligned}
$$

According to Lemma $3.11 H_{\exists}(q, F) \wedge \mathrm{SKO}_{q}(\exists x p(x)) \wedge \forall y \neg \triangle p(y)$ is 1-satisfiable if and only if $F$ is 1-satisfiable. We refer to it as the Skolemized form of $F$ with respect to satisfiability.

Although standard Skolemization does not preserve 1-satisfiability for all prenex formulas of $\mathbf{G}_{\infty}^{\triangle}$, it does so for formulas in which the quantifier free part is preceded by $\triangle$. Indeed, formulas of the form $\mathrm{Q} \bar{x} \triangle B$, where $B$ is quantifier-free, can be Skolemized in the standard way, i.e., every existentially quantified variable $x$ is replaced by a Skolem term $f(\bar{y})$, where $\bar{y}$ denotes the variables bound by universal quantifiers in the scope of which $x$ occurs (perfectly dual to Definition 3.1). We will denote by $\forall \bar{x} \triangle B^{S}$ the formula arising from $\mathrm{Q} \bar{x} \triangle B$ in this manner.

Lemma 3.13. $\mathrm{Q} \bar{x} \triangle B \in 1 \mathrm{SAT} \Longleftrightarrow \forall \bar{x} B^{S} \in 1 \mathrm{SAT}$.

Proof. $(\Leftarrow)$ Easy. For $(\Rightarrow)$ note that a formula $\exists y \triangle B^{\prime}(y)$ evaluates to 1 under an interpretation $I$ if and only if $\left\|B^{\prime}\right\|_{I[d / y]}=1$ for some domain element $d$.

This observation can be exploited to achieve a more efficient translation of conjunctions of prenex formulas, as stated in the following corollary.

Corollary 3.14. Let $F=\bigwedge_{1 \leq i \leq m} A_{i}$, where $A_{i}$ are prenex formulas of $\mathbf{G}_{\infty}$ and for $1 \leq$ $i \leq m_{1}\left(m_{1} \leq m\right) A_{i}$ is of the form $\mathrm{Q} \bar{x} \triangle B_{i}$ for some quantifier free formula $B_{i}$. Then

$$
F \in 1 \mathrm{SAT} \Longleftrightarrow\left(\bigwedge_{1 \leq i \leq m_{1}} \forall \bar{x} A_{i}^{S} \wedge H_{\exists}\left(q, \bigwedge_{m_{1} \leq i \leq m} A_{i}\right) \wedge \bigwedge_{m_{1} \leq i \leq m} \operatorname{SKO}_{q}\left(A_{i}\right)\right) \in 1 \mathrm{SAT} .
$$

\section{Theorem Proving}

Let $F$ be a conjunction of prenex formulas. The results of the last section amount to the following central "preprocessing steps" for automated theorem proving:

- Testing validity of $F$ can be reduced to testing validity of a purely existential formula $\exists \bar{x} G$ (Corollary 3.6).

- Testing 1-satisfiability of $F$ can be reduced to testing 1-satisfiability of a purely universal formula $\forall \bar{x} G^{\prime}$ (Corollary 3.14).

Note that the first problem is $\Sigma_{1}$-complete (because $\mathbf{G}_{\infty}^{\triangle}$ is recursively axiomatizable [19]), whereas the second problem is $\Pi_{1}$-complete [20], just like the corresponding problems for classical logic. However, in contrast to classical logic, the problems are not simply dual to each other: to obtain $G^{\prime}$ we even had to extend the signature of $F$ and $G$ by introducing a new predicate symbol. Nevertheless we can treat $\exists \bar{x} G$ and $\forall \bar{x} G^{\prime}$ in the same manner for our next step towards efficient theorem proving: translating the quantifier free part $\left(G, G^{\prime}\right)$ into a suitable normal form.

In our case this normal form will directly correspond to so-called order clauses that refer to the (classical) theory of dense linear orders with endpoints. In this manner both, the validity and the 1-satisfiability problem for prenex $\mathbf{G}_{\infty}^{\Delta}$, are reduced to detecting the (un)-satisfiability of specific sets of order clauses. For handling the latter problem we can 
rely on results from the literature on automated theorem proving using ordered chaining resolution, as we will point out in Section 4.2.

In fact, a particular normal form for propositional formulas of $\mathbf{G}_{\infty}^{\triangle}$, called chain normal form, has already been described in the literature, see e.g., [3, 8. To recall this notion let us use, in addition to the abreviations $\triangleleft$ and $\unlhd$ (Definition 3.9), also $A \triangleq B$ as an abbreviation for $\triangle(A \leftrightarrow B)$. Clearly $\|A \triangleq B\|_{I}=1$ iff $\|A\|_{I}=\|B\|_{I}$.

Definition 4.1. Let $F$ be a quantifier-free formula of $\mathbf{G}_{\infty}^{\triangle}$ and let $A_{1}, \ldots, A_{n}$ be the atoms occurring in $F$. A $\triangle$-chain over $F$ is a formula of the form

$$
\left(\perp \bowtie_{0} A_{\pi(1)}\right) \wedge\left(A_{\pi(1)} \bowtie_{1} A_{\pi(2)}\right) \wedge \cdots \wedge\left(A_{\pi(n-1)} \bowtie_{n-1} A_{\pi(n)}\right) \wedge\left(A_{\pi(n)} \bowtie_{n} \top\right)
$$

where $\pi$ is a permutation of $\{1, \ldots, n\}, \rtimes_{i}$ is either $\triangleleft$ or $\triangleq$, but at least one of the $\bowtie_{i}$ 's stands for $\triangleleft$.

By Chains $(F)$ we denote the set of all $\triangle$-chains over $F$.

The following follows immediately from Theorem 17 of [3].

Theorem 4.2. Let $F$ be of the form $\bigwedge_{1 \leq i \leq n} \forall \overline{x_{i}} \triangle F_{i}$, where $F_{i}$ is quantifier free. Then there exist $\Gamma_{i} \subseteq$ Chains $\left(F_{i}\right)$ for all $1 \leq i \leq n$ such that

$$
\models_{\mathbf{G}_{\infty}} F \leftrightarrow \bigwedge_{1 \leq i \leq n} \bigvee_{C \in \Gamma_{i}} C .
$$

While Theorem 4.2 can be used, in principle, to translate Skolemized formulas into a kind of disjunction normal form, the translation as well as the resulting normal form is excessively complex in general. To appreciate the problem, note that Chains $(F)$ contains a super-exponential number of different $\triangle$-chains (with respect to the length of $F$ ) in general. Clearly we need an alternative translation to normal form to obtain a practically feasible proof method. A suitable normal form is presented below.

4.1. Structural Translation to Order Clauses. It is well known from classical logic that the combinatorial explosion that may arise in any language preserving translation of arbitrary complex formulas into conjunctive normal form can be avoided by a structural translation. The latter introduces new predicate symbols to define appropriate abbreviations of subformulas, see [26, 7]. Our translation of Skolemized $\mathbf{G}_{\infty}^{\triangle}$-formulas to clausal form proceeds in an analogous manner. We consider this as a two-step process that can roughly be described as follows:

(1) The quantifier free part is efficiently reduced to a formula of an extended language, involving a conjunction of simple equivalences that introduce new predicate symbols as abbreviations for subformulas ("definitional normal form").

(2) The resulting $\mathbf{G}_{\infty}^{\triangle}$-formula is translated into a set of clauses, where the literals are of the form $s<t$ or $s \leq t$, referring to the (classical) theory of dense total orders with endpoints ("definitional clause form").

For step 1 we introduce the following notions. (Remember that negations $\neg F$ appear as $F \rightarrow \perp$.)

Definition 4.3. For any quantifier free formula $F$ of the form $F_{1} \circ F_{2}$, where $\circ \in\{\wedge, \vee, \rightarrow\}$, let

$$
\operatorname{df}(F) \stackrel{\text { def }}{=}\left[p_{F}(\bar{x}) \triangleq\left(p_{F_{1}}\left(\overline{x_{1}}\right) \circ p_{F_{2}}\left(\overline{x_{2}}\right)\right)\right]
$$


where $p_{F}, p_{F_{1}}, p_{F_{2}}$ are new predicate symbols and $\bar{x}, \overline{x_{1}}, \overline{x_{2}}$ are the tuples of variables occurring in $F, F_{1}, F_{2}$, respectively. If $F$ is of the form $\triangle F_{1}$ then

$$
\operatorname{df}(F) \stackrel{\text { def }}{=}\left[p_{F}(\bar{x}) \triangleq \triangle p_{F_{1}}\left(\overline{x_{1}}\right)\right] .
$$

If $F$ is atomic then $p_{F}(\bar{x})$ is simply an alternative denotation for $F$.

Depending on whether we are interested in 1-satisfiability or in validity we need two different normal forms based on the equivalences introduced in Definition 4.3.

Definition 4.4. Let $A$ be a quantifier free formula. The definitional normal form for 1-satisfiability is defined as

$$
\mathrm{DF}_{\text {sat }}(A) \stackrel{\text { def }}{=} \triangle p_{A}(\bar{x}) \wedge\left(\bigwedge_{F \in \operatorname{nsf}(A)} \mathrm{df}(F)\right)
$$

The definitional normal form for validity is defined as

$$
\operatorname{DF}_{\text {val }}(A) \stackrel{\text { def }}{=}\left(\bigwedge_{F \in \operatorname{nsf}(A)} \operatorname{df}(F)\right) \rightarrow \triangle p_{A}(\bar{x})
$$

In both cases, $\operatorname{nsf}(A)$ denotes the set of all non-atomic subformulas of $A, \bar{x}$ is the tuple of variables occurring in $A$, and $p_{A}$ is a new predicate symbol.

To prove the soundness of the definitional normal form for validity for existential formulas the following lemma is needed. Its proof requires Herbrand's Theorem.

Lemma 4.5. For all quantifier free formulas $A$ of $\mathbf{G}_{\infty}^{\triangle}: \models_{\mathbf{G}_{\infty}} \exists \bar{x} A \Leftrightarrow \models_{\mathbf{G}_{\infty}} \exists \bar{x} \triangle A$.

Proof. The direction from right to left is trivial. The other direction is obtained as follows:

$$
\begin{array}{rll} 
& \models_{\mathbf{G}_{\infty}} \exists \bar{x} A & \\
\Rightarrow & \models_{\mathbf{G}_{\infty}} \bigvee_{i=1}^{n} A\left(\overline{t_{i}}\right) \text { for appropriate } \overline{t_{1}}, \ldots, \overline{t_{n}} & \text { by Theorem } 3.4 \text { (Herbrand Theorem) } \\
\Rightarrow \quad \models_{\mathbf{G}_{\infty} \triangle} \triangle \bigvee_{i=1}^{n} A\left(\overline{t_{i}}\right) & \text { since } \models_{\mathbf{G}_{\infty}} F \text { iff } \models_{\mathbf{G}_{\infty}} \triangle F \\
\Rightarrow \quad \models_{\mathbf{G}_{\infty}} \bigvee_{i=1}^{n} \triangle A\left(\overline{t_{i}}\right) & \text { by Lemma } 2.5(2) \\
\Rightarrow \quad \models_{\mathbf{G}_{\infty}} \exists \bar{x} \triangle A(\bar{x}) & \text { by laws of } \mathbf{G}_{\infty}^{\triangle} .
\end{array}
$$

Note that in the second and in the last step we used the fact that for quantifier free $A(\bar{x})$ the formula $\exists \bar{x} A(\bar{x})$ is already in Skolem form.

We remark that the above lemma does not hold when $A$ is not quantifier free.

Lemma 4.6. Let $A$ be a quantifier free formula with free variables $\bar{x}$.

(a) $\forall \bar{x} A$ is 1-satisfiable iff $\forall \bar{x} \mathrm{DF}_{\text {sat }}(A)$ is 1-satisfiable.

(b) $\exists \bar{x} A$ is valid iff $\exists \bar{x} \mathrm{DF}_{\text {val }}(A)$ is valid.

Proof. Note that all the relevant subformulas of $\mathrm{DF}_{\text {sat }}(A)$ and $\mathrm{DF}_{\text {val }}(A)$ are preceded by an occurrence of $\triangle$. Thus we obtain in the same manner as for the definitional normal forms of classical logic $\left(\right.$ see [26, 7]) that $\operatorname{val}_{I}(\operatorname{df}(F(\bar{x})))=1$ iff $\operatorname{val}_{I}(F(\bar{x}))=\operatorname{val}_{I}\left(p_{F}(\bar{x})\right)$ for all subformulas $F$ of $A$. Consequently we have:

$\left(*_{1}\right)$ Every model of $A$ can be extended to a model of $\operatorname{DF}_{\text {sat }}(A)$; conversely every model of $\mathrm{DF}_{\text {sat }}(A)$ is also a model of $A$.

$\left(*_{2}\right)$ All extensions of a model of $A$ to the language that additionally contains the new predicate symbols $p_{F}$ for $F \in \operatorname{nsf}(A)$ are models of $\mathrm{DF}_{\text {val }}(A)$; conversely every model $I$ of $\mathrm{DF}_{\text {val }}(A)$ where $\operatorname{val}_{I}\left(p_{A}(\bar{x})\right)=1$ is also a model of $A$. 
To obtain (a) from $\left(*_{1}\right)$ it suffices to remember that, for arbitrary formulas $F, \forall x F$ is 1-satisfiable iff $F$ there is an interpretation $I$ such that $I[\bar{d} / \bar{x}]$ is a model of $F$ for every variable assignment $[\bar{d} / \bar{x}]$.

To obtain (b) something more is needed, since in general an interpretation $v_{I}$ can be a model for $\exists x F$ even if $\|F(x)\|_{I[d / x]}<1$ for each variable assignment. However note that this cannot happen if $F$ is of the form $\triangle G$. More precisely:

$\left(*_{3}\right)$ For all fomulas $G, \models_{\mathbf{G}_{\infty}} \exists \bar{x} \triangle G$ iff for every interpretation $I$ there is a variable assignment $[\bar{d} / \bar{x}]$ such that $\|G(\bar{x})\|_{I[\bar{d} / \bar{x}]}=1$.

Therefore, using Lemma 4.5, we argue as follows:

$$
\begin{array}{rlr} 
& \models_{\mathbf{G}_{\infty}} \exists \bar{x} A & \\
\Leftrightarrow & \models_{\mathbf{G}_{\infty}} \exists \bar{x} \triangle A & \text { by Lemma 4.5 } \\
\Leftrightarrow & \models_{\mathbf{G}_{\infty}} \exists \bar{x} \triangle \mathrm{DF}_{\text {val }}(A) & \text { by }\left(*_{2}\right) \text { and }\left(*_{3}\right) \\
\Leftrightarrow & \models_{\mathbf{G}_{\infty}} \exists \bar{x} \mathrm{DF}_{\text {val }}(A) & \text { by Lemma 4.5 }
\end{array}
$$

We now switch to step 2 of the translation into clausal form, which results in a "logic free" syntax by considering all predicate symbols as function symbols and the special atomic formulas $T$ and $\perp$ as constant symbols. More precisely, an atomic $\mathbf{G}_{\infty}{ }_{\infty}$-formula like $p(x, f(x, y))$ will no longer be considered to be a formula of the new language, but now simply appears as a term containing two binary function symbols. We will use $\mathbf{T}$ to denote the set of all terms arising in this manner.

Definition 4.7. An order literal is an expression of the form $s<t$ or $s \leq t$, where $s, t \in \mathbf{T}$. An order clause is a finite set of literals, representing a disjunction of its elements 6

Semantically order clauses refer to the following classical structure.

Definition 4.8. By a dense total order $O$ we mean an interpretation of the predicate symbols $<$ and $\leq$, taking terms in $\mathbf{T}$ as arguments, where $<$ refers to a strict and dense total (linear) order and $\leq$ is interpreted as the reflexive closure of $<$. If, in addition, the endpoint axioms $\forall x(\perp \leq x), \forall x(x \leq \top)$, and $\perp<\top$ are satisfied we call $O$ a DTOE-model.

A set of order clauses $S$ is DTOE-satisfiable if the conjunction of elements of $S$ has a dense total order with endpoints $\perp$ and $T$ as a model; otherwise $S$ is called DTOEunsatisfiable.

The normal form that will be used in the next section is a translation of the definitional normal forms in Definition 4.4 into suitable order clauses.

Definition 4.9. Let $A, B$, and $C$ be atomic formulas.

$$
\begin{array}{lll}
\operatorname{cl}(C \triangleq(A \wedge B)) & \stackrel{\text { def }}{=} \quad\{\{C \leq A\},\{C \leq B\},\{A \leq C, B \leq C\}\} \\
\operatorname{cl}(C \triangleq(A \vee B)) & \stackrel{\text { def }}{=} \quad\{\{A \leq C\},\{B \leq C\},\{C \leq A, C \leq B\}\} \\
\operatorname{cl}(C \triangleq(A \rightarrow B)) \stackrel{\text { def }}{=} \quad\{\{A \leq B, C \leq B\},\{\top \leq C, B<A\}, \\
\\
\operatorname{cl}(C \triangleq \triangle A) & \stackrel{\text { def }}{=} \quad\{\{C \leq \perp, \top \leq A\},\{\top \leq C, A<\top\}\}
\end{array}
$$

\footnotetext{
${ }^{6}$ In [11] order clauses are defined as multisets. However it follows from results in [1] concerning redundancy that we may alternatively define clauses as sets.
} 
For a quantifier free formula $G$ the definitional clause form for 1-satisfiability is defined as

$$
\left.\mathrm{CF}_{\text {sat }}(G) \stackrel{\text { def }}{=}\left\{\left\{\top \leq p_{G}(\bar{x})\right\}\right\} \cup \underset{F \in \operatorname{nsf}(G)}{\bigcup} \operatorname{cl}(\operatorname{df}(F))\right)
$$

and the definitional clause form for validity is defined as

$$
\left.\mathrm{CF}_{\text {val }}(G) \stackrel{\text { def }}{=}\left\{\left\{p_{G}(\bar{x})<\top\right\}\right\} \cup \underset{F \in \operatorname{nsf}(G)}{\bigcup} \operatorname{cl}(\operatorname{df}(F))\right),
$$

where $\operatorname{nsf}(G)$ denotes the set of all non-atomic subformulas of $G, \bar{x}$ is the tuple of variables occurring in $G$, and $p_{G}$ is a new predicate symbol.

Lemma 4.10. Let $A$ be a quantifier free formula with free variables $\bar{x}$.

(a) $\forall \bar{x} \mathrm{DF}_{\text {sat }}(A)$ is 1-satisfiable iff $\mathrm{CF}_{\text {sat }}(A)$ is DTOE-satisfiable.

(b) $\exists \bar{x} \mathrm{DF}_{\text {val }}(A)$ is valid iff $\mathrm{CF}_{\mathrm{val}}(A)$ is $\mathrm{DTOE}$-unsatisfiable.

Proof. We have to check that the clauses specified in Definition 4.9 are equivalent to the corresponding subformulas involving ' $\triangleq$ ' in the definitional forms specified in Definition 4.4. - $C \triangleq(A \rightarrow B)$ : it is not difficult to see that the following formulas are equivalent in $\mathbf{G}_{\infty}^{\triangle}$, i.e. for each interpretation $I$ we have

$$
\|\triangle(C \leftrightarrow(A \rightarrow B))\|_{I}=\|(A \unlhd B \wedge \triangle C) \vee(B \triangleleft A \wedge C \unlhd B \wedge B \unlhd C)\|_{I} .
$$

By applying the law of distribution to the formula at the right hand side we obtain the conjunction of the following six formulas (note that we can express $\triangle C$ by the equivalent formula $\top \unlhd C$, cf. Definition (3.9):

$$
\begin{array}{lll}
A \unlhd B & \vee & B \triangleleft A \\
A \unlhd B & \vee & C \unlhd B \\
A \unlhd B & \vee & B \unlhd C \\
\top \unlhd & \vee & B \triangleleft A \\
\top \unlhd C & \vee & C \unlhd B \\
\top \unlhd & \vee & B \unlhd C
\end{array}
$$

Note that conjunct (1) is valid and that $B \unlhd C$ is entailed by (6). $B \unlhd C$ in turn entails conjuncts (3) and (6). Thus we obtain the following four conjuncts that directly correspond to $\operatorname{cl}(C \triangleq(A \rightarrow B))$ :

$$
\begin{aligned}
& A \unlhd B \vee C \unlhd B \\
& \top \unlhd C \vee B \triangleleft A \\
& \top \unlhd C \vee C \unlhd B \\
& B \unlhd C
\end{aligned}
$$

- $C \triangleq(A \wedge B): \triangle(C \leftrightarrow(A \wedge B))$ is easily seen to be equivalent to the conjunction of

$$
\begin{aligned}
& C \unlhd A \\
& C \unlhd B \\
& A \unlhd C \vee B \quad B \unlhd C
\end{aligned}
$$

that directly correspond to $\operatorname{cl}(C \triangleq(A \wedge B))$. 
- $C \triangleq(A \vee B): \triangle(C \leftrightarrow(A \vee B))$ is equivalent to the conjunction of

$$
\begin{aligned}
& A \unlhd C \\
& B \unlhd C \\
& C \unlhd A \vee C \unlhd B
\end{aligned}
$$

that directly correspond to $\operatorname{cl}(C \triangleq(A \vee B))$.

- $C \triangleq \triangle A: \triangle(C \leftrightarrow \triangle A)$ is equivalent to the conjunctions of the following two disjunctions

$$
\begin{array}{lll}
C \unlhd & \vee & \top \unlhd A \\
\top \unlhd & \vee & A \triangleleft \top
\end{array}
$$

that directly correspond to $\operatorname{cl}(C \triangleq \triangle A)$.

So far we have argued about equivalences within $\mathbf{G}_{\infty}^{\triangle}$. But note that formulas of the form $A \unlhd B$ or $A \triangleleft B$ evaluate to either 0 or 1 in every interpretation. Therefore disjunction and conjunction reduce to their classical counterparts and we can directly translate $A \unlhd B$ and $A \triangleleft B$ into order literals $A \leq B$ and $A<B$, respectively. In this manner we obtain sets of order clauses that are DTOE-satisfiable iff the corresponding $\mathbf{G}_{\infty}^{\triangle}$-formulas are 1-satisfiable. In the case of $\mathrm{CF}_{\text {sat }}(A)$ the clause $\left\{\top \leq p_{G}(\bar{x})\right\}$ directly codes the claim that $A \in$ 1SAT, whereas for $\mathrm{CF}_{\text {val }}(A)$ the clause $\left\{p_{G}(\bar{x})<1\right\}$ ensures that $A$ is valid iff $\mathrm{CF}_{\text {val }}(A)$ is DTOEunsatisfiable.

Remark 4.11. A somewhat different structural clause form has been described in [3]. Here, following [8], we have eliminated a number of redundancies from the originally described sets of order clauses.

The following theorem combines the various steps of the current and the last section and points out the efficiency of the overall translation.

Theorem 4.12. Let $A=\bigwedge_{1 \leq i \leq m} A_{i}$ where $A_{i}$ are prenex formulas of $\mathbf{G}_{\infty}{ }_{\infty}$. Then one can construct in polynomial time sets of order clauses $S_{\text {sat }}(A)$ and $S_{\text {val }}\left(A_{i}\right)$, where $1 \leq i \leq n$, such that

(a) $A \in 1 \mathrm{SAT}$ iff $S_{\text {sat }}(A)$ is DTOE-satisfiable.

(b) $\models_{\mathbf{G}_{\infty}} A$ iff $S_{\text {val }}\left(A_{i}\right)$ is DTOE-unsatisfiable for each $i \in\{1, \ldots, n\}$.

Proof. (a) By Corollary 3.14 we obtain a formula $B=\bigwedge_{1 \leq i \leq n} \forall \bar{x}_{i} B_{i}$, where the $B_{i}$ are quantifier free, such that $A \in 1 \mathrm{SAT}$ iff $B \in 1 \mathrm{SAT}$. Remember that the conjunction of formulas corresponds to the union of sets of clauses. Therefore the combination of Lemmas 4.6 and 4.10 implies that $A$ is 1-satisfiable iff $S_{\text {sat }}(A)=\bigcup_{1 \leq i \leq n} \mathrm{CF}_{\text {sat }}\left(B_{i}\right)$ is DTOE-satisfiable.

(b) By Corollary 3.6 we obtain a formula $B=\bigwedge_{1<i \leq n} \exists \bar{x}_{i} B_{i}$, where the $B_{i}$ are quantifier free, such that $\models_{\mathbf{G}_{\infty}} A$ iff $\models_{\mathbf{G}_{\infty}} B$. Since a conjunction is valid iff every conjunct is valid, Lemmas 4.6 and 4.10 reduce the problem of checking whether $\models_{\mathbf{G}_{\infty}} A$ to checking whether for each $i \in\{1, \ldots, n\}$ the clause set $S_{\text {val }}\left(A_{i}\right)=\mathrm{CF}_{\text {val }}\left(B_{i}\right)$ is DTOE-unsatisfiable.

It finally remains to observe that $S_{\text {sat }}(A)$ and the $S_{\text {val }}\left(A_{i}\right)$ are of polynomial size with respect to the size of $A$. In particular note that $H_{\exists}(q, A)$ (Definition 3.10) and $\operatorname{SKO}_{q}\left(A_{i}\right)$ (Definition 3.7) increase the overall size of the formula only by a linear number of symbols. Also the definitional clause forms (Definition 4.9) are linear in the size of $A$. Consequently, all mentioned transformations can clearly be done in polynomial time. 
Example 4.13. We claim that the following formula is valid in $\mathbf{G}_{\infty}^{\triangle}$ :

$$
A=\exists x \forall y(\triangle p(y) \rightarrow p(x)) .
$$

While simple, this example is nevertheless of some interest. In particular note that removing the occurrence of $\triangle$ in $A$ results in a formula that is not any longer valid in $\mathbf{G}_{\infty}^{\triangle}$, although it is classically valid.

According to Section 3.1 we obtain the Skolemized form of $A$ as

$$
\exists x(\triangle p(f(x)) \rightarrow p(x)) .
$$

To compute the definitional normal form we have to introduce the following two "definitions" of subformulas as described in Definition 4.3,

$\operatorname{df}\left(\triangle p(f(x)): p_{1}(x) \triangleq \triangle p(f(x))\right.$, $\operatorname{df}\left(p_{1}(x) \rightarrow p(x)\right): p_{2}(x) \triangleq\left(p_{1}(x) \rightarrow p(x)\right)$.

The corresponding order clauses according to Definition 4.9 are as follows:

$\operatorname{cl}\left(p_{1}(x) \triangleq \triangle p(f(x))\right.$ :

$1:\left\{p_{1}(x) \leq \perp, \top \leq p(f(x))\right\}$

$2:\left\{\top \leq p_{1}(x), p(f(x))<\top\right\}$

$\operatorname{cl}\left(p_{2}(x) \triangleq\left(p_{1}(x) \rightarrow p(x)\right)\right.$ :

$3:\left\{p_{1}(x) \leq p(x), p_{2}(x) \leq p(x)\right\}$

$4:\left\{\top \leq p_{2}(x), p(x)<p_{1}(x)\right\}$

$5:\left\{\top \leq p_{2}(x), p_{2}(x) \leq p(x)\right\}$

$6:\left\{p(x) \leq p_{2}(x)\right\}$

Since we are interested in validity we have to add

$7:\left\{p_{2}(x)<\top\right\}$

to obtain $\mathrm{CF}_{\text {val }}(\triangle p(f(x)) \rightarrow p(x))$ as specified in Definition 4.9 .

We will continue this example in Section 4.2 to illustrate a machine oriented proof of the DTOE-unsatisfiability of $\mathrm{CF}_{\text {val }}(\triangle p(f(x)) \rightarrow p(x))$.

4.2. Ordered Chaining Resolution. The results of the previous sections, as summarized in Theorem 4.12, reduce the validity as well as the 1-satisfiability problem for prenex $\mathbf{G}_{\infty}^{\triangle}$ to checking DTOE-(un)satisfiability of certain sets of order clauses. Fortunately, efficient theorem proving for various types of order clauses has already received considerable attention in the literature; see [12, 11] and the references given there. We finally just extract from this literature what is needed in our specific case.

We recall some basic notions from automated deduction (see, e.g., 23]). In particular we identify a substitution $\sigma$ with a set $\left\{x_{1} \leftarrow t_{1}, \ldots, x_{n} \leftarrow t_{n}\right\}$ and define $\operatorname{codom}(\sigma)=$ $\left\{t_{1}, \ldots, t_{n}\right\}$. $E \sigma$ denotes the result of applying $\sigma$ to an expression $E$, i.e. $E \sigma$ is obtained by replacing for each $i \in\{1, \ldots, n\}$ all occurrences of the variable $x_{i}$ in $E$ by the term $t_{i}$. Finally, recall that a substitution $\sigma$ is called the most general unifier (mgu) of terms $s_{1}, \ldots, s_{n}$ if $s_{1} \sigma=\ldots=s_{n} \sigma$ and if in addition for all other substitutions $\rho$ where $s_{1} \rho=$ $\ldots=s_{n} \rho$ we have $s_{i} \rho=\left(s_{i} \sigma\right) \tau$ for some substitution $\tau$.

We consider the following rules (cf. [11]) for order clauses:

Irreflexivity Resolution:

$$
\frac{C \cup\{s<t\}}{C \sigma}
$$

where $\sigma$ is the mgu of $s$ and $t$ 


\section{(Factorized) Chaining:}

$$
\frac{C \cup\left\{u_{1} \triangleleft_{1} s_{1}, \ldots, u_{m} \triangleleft_{m} s_{m}\right\} \quad D \cup\left\{t_{1} \triangleleft_{1}^{\prime} r_{1}, \ldots, t_{n} \triangleleft_{n}^{\prime} r_{n}\right\}}{C \sigma \cup D \sigma \cup\left\{u_{i} \sigma \triangleleft_{i, j} r_{j} \sigma \mid 1 \leq i \leq m, 1 \leq n\right\}}
$$

where $\sigma$ is the mgu of $s_{1}, \ldots, s_{m}, t_{1}, \ldots, t_{n}$ and $\triangleleft_{i, j}$ is $<$ if and only if either $\triangleleft_{i}$ is $<$ or $\triangleleft_{j}^{\prime}$ is $<$. Moreover, $t_{1} \sigma$ occurs in $D \sigma$ only in inequalities $r^{6} \triangleleft t_{1} \sigma$.

These two rules constitute a refutationally complete inference system for the theory of all total orders in presence of set $\mathbf{E} \mathbf{q}^{\mathbf{F}}$ of clauses

$$
\left\{x_{i}<y_{i}, y_{i}<x_{i} \mid 1 \leq i \leq n\right\} \cup\left\{f\left(x_{1}, \ldots, x_{n}\right) \leq f\left(y_{1}, \ldots, y_{n}\right)\right\},
$$

where $f$ ranges over the set $\mathbf{F}$ of function symbols of the signature. Observe that, in translating a formula $P$ from prenex $\mathbf{G}_{\infty}^{\triangle}$ into a set of order clauses $\mathrm{CF}_{\text {sat }}(P)$, we treat the predicate symbols of $P$ as function symbols. Additional function symbols occur from Skolemization.

The inference system is not yet sufficiently restrictive for efficient proof search. We follow [11] and add conditions to the rules that refer to some complete reduction order $\succ$ (on the set of all terms). We write $s \nsucceq t$ if $\neg(s \succ t)$ and $s \neq t$; and " $t$ is basic in (clause) $C$ " if $t \triangleleft s \in C$ or $s \triangleleft t \in C$.

Maximality Condition for Irreflexivity Resolution: $s \sigma$ is a maximal term in $C \sigma$. Maximality Condition for Chaining:

(1) $u_{i} \sigma \nsucceq s_{1} \sigma$ for all $1 \leq i \leq n$,

(2) $v_{i} \sigma \nsucceq t_{1} \sigma$ for all $1 \leq i \leq m$,

(3) $u \sigma \nsucceq s_{1} \sigma$ for all terms $u$ that are basic in $C$, and

(4) $v \sigma \nsucceq t_{1} \sigma$ for all terms $v$ that are basic in $D$.

For our purposes it is convenient to view the resulting inference system $\mathrm{MC}_{\succ}$ as a set operator.

Definition 4.14. $\mathrm{MC}_{\succ}(S)$ is the set of all conclusions of Irreflexivity Resolution or Maximal Chaining where the premises are (variable renamed copies of) members of the set of clauses $S$. Moreover, $\operatorname{MC}_{\succ}^{0}(S)=S, \operatorname{MC}_{\succ}^{i+1}(S)=\operatorname{MC}_{\succ}\left(\operatorname{MC}_{\succ}^{i}(S)\right) \cup \operatorname{MC}_{\succ}^{i}(S)$, and $\operatorname{MC}_{\succ}^{*}(S)=\bigcup_{i \geq 0} \operatorname{MC}_{\succ}^{i}(S)$.

The set consisting of the three clauses $\{\perp \leq y\},\{y \leq \top\}$, and $\{\perp<\top\}$, corresponding to the endpoint axioms, is called Ep. The set consisting of $\{y \leq x, d(x, y)<y\}$ and $\{y \leq x, x<d(x, y)\}$, corresponding to the usual density axiom, is called Do.

The following completeness theorem follows directly from Theorem 2 of [1].

Theorem 4.15. $S$ has a dense total order with endpoints 0 and 1 as a model if and only if $\operatorname{MC}_{\succ}^{*}(S \cup \mathbf{E q} \mathbf{F} \cup \mathbf{E p} \cup \mathbf{D o})$ does not contain the empty clause.

Remark 4.16. Even more refined "chaining calculi" for handling orders have been defined by Bachmair and Ganzinger in [11, 12]. However, $\mathrm{MC}_{\succ}$ turns out to be quite appropriate for our context. (In particular, since the problem of "variable chaining" does not occur for the sets of clauses considered here).

Example 4.17. (Example 4.13 continued) According to Theorem 4.15 we should add the sets of clauses $\mathbf{E} \mathbf{q}^{\mathbf{F}}, \mathbf{E p}$, and Do to $\mathrm{CF}_{\text {val }}(\triangle p(f(x)) \rightarrow p(x))$, in order to guarantee that Irreflexivity Resolution and Chaining suffice to derive the empty clause, witnessing the validity of $\exists x(\triangle p(f(x) \rightarrow p(x))$ and consequently also of $\exists x \forall y(\triangle p(y) \rightarrow p(x))$. However it turns out that only the following subset of clauses is actually needed for this purpose: 


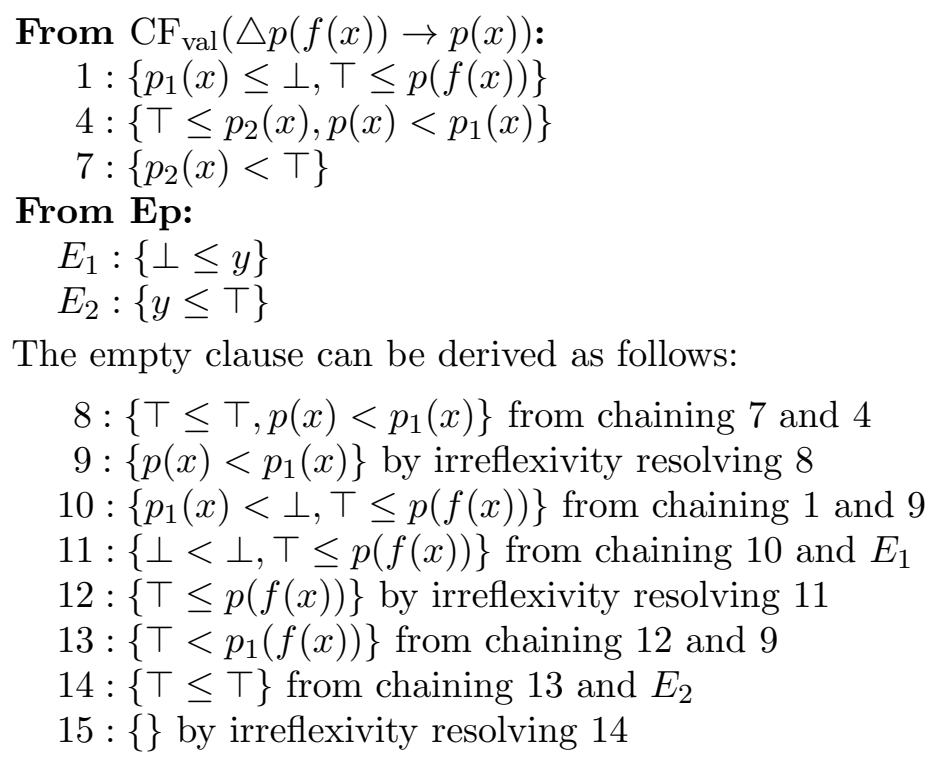

\section{Conclusion}

We took up the challenge of providing logical foundations for efficient theorem proving for $\mathbf{G}_{\infty}^{\triangle}$, i.e., Gödel logic augmented by the projection operator $\triangle$. In contrast to classical logic, testing validity of a $\mathbf{G}_{\infty}^{\triangle}$-formula $F$ is not equivalent to testing the (1-)unsatisfiability of $\neg F$. However both problems are important in view of intended applications. Unfortunately, efficient proof search methods for unrestricted first-order $\mathbf{G}_{\infty}^{\triangle}$ seem, at least currently, to be out of reach. In particular, Skolemization for full $\mathbf{G}_{\infty}^{\triangle}$, even without $\triangle$, is an open problem. Consequently, we have focused on the (still very expressive) prenex fragment of $\mathbf{G}_{\infty}^{\triangle}$ and described a proof search method that remains as close as possible in spirit to resolution based theorem proving for classical logic. In particular this allows us to treat both problems, testing validity and testing 1-unsatisfiability, in a uniform manner. While, as we have shown by proving a version of Herbrand's theorem, standard Skolemization preserves validity for prenex $\mathbf{G}_{\infty}^{\triangle}$, we had to come up with a novel, extended form of Skolemization for satisfiability. In both cases, Skolemized formulas are efficiently translated into a specific structural normal form. This consists of sets of order clauses, where the literals are of the from $s<t$ or $s \leq t$. We have finally explained how chaining resolution, a well investigated proof search method for order clauses, can be employed to check unsatisfiability in a machine oriented manner.

We like to emphasize that our results not only provide a basis for automated proof search, but also demonstrate a number of interesting logical properties of $\mathbf{G}_{\infty}^{\triangle}$ that distinguish it, e.g., from other fuzzy logics [18]. For example, the fact that the set of 1-unsatisfiable prenex formulas of $\mathbf{G}_{\infty}^{\triangle}$ is recursively enumerable (as trivially implied by our results) has not been known previously. 


\section{ACKNOWLEDGEMENT}

We thank the referees of this paper and Daniel Weller for valuable remarks that lead to considerable improvements.

\section{REFERENCES}

[1] A. Avron. Hypersequents, logical consequence and intermediate logics for concurrency. Annals of Mathematics and Artificial Intelligence 4: 225-248, 1991.

[2] M. Baaz. Infinite-valued Gödel logics with 0-1-projections and relativizations. In Proceedings Gödel 96. Kurt Gödel's Legacy. Springer LNL 6, 23-33, 1996.

[3] M. Baaz, A. Ciabattoni and C.G. Fermüller. Herbrand's Theorem for prenex Gödel logic and its consequences for theorem proving. Proceedings of LPAR'2001. Springer LNAI 2250, 201-216, 2001.

[4] M. Baaz, A. Ciabattoni and C.G. Fermüller. Hypersequent calculi for Gödel Logics — a survey. J. of Logic and Computation 13, 1-27, 2003.

[5] M. Baaz, A. Ciabattoni and N. Preining. SAT in monadic Gödel logics: a borderline between decidability and undecidability. Proceedings of WOLLIC 2009. Springer LNAI 5514, 113-123, 2009.

[6] M. Baaz, S. Hetzl and D. Weller. On the complexity of proof deskolemization. J. Symbolic Logic. To appear.

[7] M. Baaz, U. Egly and A. Leitsch. Normal form transformations. Handbook of Automated Reasoning, Vol. 1. Eds: A. Robinson, A. Voronkov. Elsevier, 2001, 273-333.

[8] M. Baaz and C.G. Fermüller. A resolution mechanism for prenex Gödel logic. In Proceedings of CSL 2010. Springer LNCS 6247, 67-79, 2010.

[9] M. Baaz and N. Preining. Gödel-Dummett logics. Handbook of Mathematical Fuzzy Logic, volume 2, Eds: P. Cintula, P. Hájek, C. Noguera. College Publications, pp. 585-627, 2011.

[10] M. Baaz, N. Preining and R. Zach. First-order Gödel logics. Annals of Pure and Applied Logic 147:23-47, 2007.

[11] L. Bachmair and H. Ganzinger. Ordered chaining for total orderings. Proc. CADE`94, Springer LNCS 814, 1994, 435-450.

[12] L. Bachmair and H. Ganzinger. Ordered chaining calculi for first-order theories of transitive relations. J. ACM 45(6): 1007-1049, 1998.

[13] A. Ciabattoni and P. Rusnok. On the classical content of monadic $\mathrm{G}^{\sim}$ and its application to a fuzzy medical expert system, Proceedings of KR 2010, AAAI, 373-381, 2010.

[14] A. Degtyarev and A. Voronkov. Decidability problems for the prenex fragment of intuitionistic logic. In Proceedings LICS'96. IEEE Press, 503-509, 1996.

[15] M. Dummett. A propositional calculus with denumerable matrix. J. Symbolic Logic 24: 97-106, 1959.

[16] D.M. Gabbay. Decidability of some intuitionistic predicate theories. J. of Symbolic Logic 37: 579-587, 1972.

[17] G. Fiorino. Fast decision procedure for propositional Dummett logic based on a multiple premise tableau calculus. Inf. Sci. 180(19): 3633-3646, 2010.

[18] P. Hájek. Metamathematics of Fuzzy Logic. Kluwer, 1998.

[19] P. Hájek. Arithmetical complexity of fuzzy predicate logics - a survey. Soft Computing 9/12: 935-941, 2005.

[20] P. Hájek. Arithmetical complexity of fuzzy predicate logics - a survey II. Annals of Pure and Applied Logic 161(2): 212-219, 2009.

[21] D. Hilbert and P. Bernays. Grundlagen der Mathematik, Vol. 2, Berlin: Springer 1939.

[22] D. Larchey-Wendling. Graph-based decision for Gödel-Dummett logics. J. Autom. Reasoning 38(1-3): 201-225, 2007.

[23] A. Leitsch. The Resolution Calculus. Springer (fomerly Kluwer) 1997.

[24] V. Lifschitz, D. Pearce and A. Valverde. Strongly equivalent logic programs. ACM Transaction on Computational Logic, 2(4): 526-541, 2001.

[25] G. Mints. The Skolem method in intuitionistic calculi. Proceedings of Steklov Institute of Mathematics, 121: 73-109, 1972.

[26] D. Plaisted and S. Greenbaum. A structure-preserving clause form translation. J. Symbolic Computation 2: 293-304, 1986. 
[27] N. Preining. Gödel logics - a survey. Proceedings of LPAR 2010. Springer LNCS 6397, pp. 30-51, 2010.

[28] G. Takeuti and T. Titani. Intuitionistic fuzzy logic and intuitionistic fuzzy set theory. J. of Symbolic Logic, 49: 851-866, 1984.

[29] A. Visser. On the completeness principle: a study of provability in Heyting's Arithmetic. Annals of Math. Logic 22: 263-295, 1982.

This work is licensed under the Creative Commons Attribution-NoDerivs License. To view a copy of this license, visit http://creativecommons.org/licenses/by-nd/2.0/ or send a letter to Creative Commons, 171 Second St, Suite 300, San Francisco, CA 94105, USA, or Eisenacher Strasse 2, 10777 Berlin, Germany 